\title{
Numerical investigation of the interaction of a vortex dipole with a deformable plate
}

\author{
Eugene Zivkov ${ }^{\mathrm{a}}$, Serhiy Yarusevych ${ }^{\mathrm{a}}$, Maurizio Porfiri ${ }^{\mathrm{b}}$, Sean D. Peterson, ${ }^{\mathrm{a}, *}$ \\ ${ }^{a}$ Department of Mechanical and Mechatronics Engineering, University of Waterloo, \\ Waterloo, Canada \\ ${ }^{b}$ Department of Mechanical and Aerospace Engineering, New York University \\ Polytechnic School of Engineering, New York, USA
}

\begin{abstract}
Energy harvesting from coherent fluid structures is a current research topic as it pertains to the design of small self-powered sensors for underwater applications. The impact of a vortex dipole with a deformable cantilevered plate at the plate tip is herein studied numerically using a strongly coupled staggered fluid-structure interaction algorithm. Three dipole Reynolds numbers, $\mathrm{Re}=500,1500$, and 3000, are investigated for constant plate properties. As the dipole approaches the plate, positive vorticity is produced on the impact face, while negative vorticity is produced at the tip of the plate. Upon impact, the dipole splits in two and two secondary dipoles are formed. The circulation and, therefore, the trajectories of these dipoles depends on both the Reynolds number and the elasticity of the plate, and these secondary dipoles may return for subsequent impacts. While the maximum deflection of the plate does not depend significantly on Reynolds number, the plate response due to subsequent impacts of secondary dipoles does vary with Reynolds number. These results elucidate the strong interdependency between plate deformation and vortex dynamics, as well as the effect of Reynolds number on both.
\end{abstract}

Keywords:

computational fluid dynamics, energy harvesting, fluid-structure interaction, Lamb dipole, vortex dynamics

\footnotetext{
*Corresponding author

Email address: peterson@mme.uwaterloo.ca (Sean D. Peterson)

$U R L$ : http://www.sdpeterson.uwaterloo.ca (Sean D. Peterson)
} 


\section{Introduction}

The desire for sensor systems and networks with long operational lives for use in remote locations has driven research into small-scale energy harvesting from the surrounding fluid. Examples include energy harvesting from flapping flags (Giacomello and Porfiri, 2011; Michelin and Doare, 2013), miniature water turbines (Myers et al., 2007; Priya et al., 2005; Cellini et al., 2014), unsteady aerodynamic loads on airfoils (De Marqui Jr. and Erturk, 2012) and the wake of bluff bodies (Allen and Smits, 2001; Akaydin et al., 2012). One particular energy harvesting modality receiving attention recently is associated with the energy exchange between coherent fluid structures and deformable plates (Akaydin et al., 2010a,b; Weinstein et al., 2012; Peterson and Porfiri, 2012b; Goushcha et al., 2014). Employing an electro-active polymer as the deformable plate facilitates conversion of mechanical energy imparted from the fluid to the plate into usable electrical energy (Nemat-Nasser and Li, 2000; Shahinpoor and Kim, 2001).

Due to their prevalence in nature and simplicity of generation in a laboratory environment, vortex rings are often employed as a canonical coherent fluid structure in energy harvesting studies involving compliant smart materials. For example, Peterson and Porfiri (2012b) presented a series of experiments in which a vortex ring impacts the tip of a cantilevered electro-active polymer and assessed the resulting energy harvesting capacity. Goushcha et al. (2014) reported on an alternate configuration in which a vortex ring advects past a cantilevered electro-active polymer plate oriented in the same direction as the ring propagation. As the ring passes by, the induced pressure distribution along the plate changes and the plate starts vibrating. Subsequent rings can either enhance or mitigate the vibration amplitude and, consequently, the energy harvesting capacity.

A first order model approximating a vortex ring approaching a wall can be constructed using potential flow by replacing the vortex ring with a pair of free vortices. The kinematics of the impact of the vortex pair with an infinite rigid wall in an ideal fluid is well known (Milne-Thomson, 1996). Saffman (1979) extended the canonical solution for point vortices to account for finite core size and demonstrated that the core deforms when the pair is in the vicinity of the wall. van Heijst and Flor (1989) presented experimental results of a dipole impacting a rigid wall. They observed a rebound effect, 
in which secondary dipoles form after the initial impact. Peace and Riley (1983), as well as Lim et al. (1991), have shown that the rebounding is in essence a viscous effect in which the pressure field of the impacting dipole results in vorticity production on the surface of the no-slip wall, which ejects and combines with the primary dipole to generate a pair of secondary dipoles that can propagate away from the wall in some cases.

Orlandi (1990) presented high Reynolds numbers numerical simulations that demonstrate increasingly complex vortex dynamics produced with increasing Reynolds number, including collisions of secondary dipoles with the wall and the formation of finer tertiary structures. The dynamics of oblique dipole/wall collisions were studied by Clercx and Bruneau (2006). Peterson and Porfiri (2013) considered the impact of a vortex dipole with the tip of a semi-infinite rigid plate. Their results show that secondary (and tertiary) dipoles form along the plate in a manner similar to the findings of Orlandi (1990), while vorticity shed from the tip combines with half of the initial dipole to generate a secondary tip dipole. The hydrodynamic loading in the vicinity of the tip of the plate during primary impact is a relatively weak function of the incoming dipole Reynolds number in the range of 500 to 3000.

Compared to previous research involving rigid boundaries, the interaction of a vortex dipole (or pair) with a compliant surface has not been investigated in detail. Peterson and Porfiri (2012a) presented a fully-coupled analytical fluid-structure interaction (FSI) model of the impact of a point-vortex pair with a deformable cantilevered plate in an ideal fluid. Their model represents the plate and vortex motion well only when the vortex pair is sufficiently far from the plate; however, the absence of viscosity precludes vorticity production along the plate. As shown in the study of the impact with a semi-infinite rigid plate (Peterson and Porfiri, 2013), the production of vorticity on the plate surface can dramatically influence the overall dynamics during impact. The present investigation considers the impact of a vortex dipole with the tip of a deformable cantilevered plate. Based on the findings from the analytical potential flow fluid-structure interaction model of Peterson and Porfiri (2012a) and their follow-up work of a dipole in a real fluid impacting a semiinfinite rigid plate (Peterson and Porfiri, 2013), the model proposed herein incorporates viscous effects to adequately resolve fluid-structure interactions and the attendant vortex dynamics. Specifically, a strongly coupled staggered FSI simulation is used to investigate the interaction of a Lamb dipole (Lamb, 1932) with a finite length cantilevered plate. The results of the simulation are analyzed in terms of vortex dynamics and plate loading, as well 
as energy transfer from the dipole into potential and kinetic energy of the plate.

\section{Problem Setup}

A schematic of the two-dimensional (2D) problem under consideration is shown in Figure 1. A Lamb dipole is initialized at a distance $d$ from a cantilevered plate of length $L$ and thickness $h$. The fluid is modeled as incompressible and Newtonian, with density $\rho_{f}$ and kinematic viscosity $\nu$. A Cartesian coordinate system is defined at the mid-point between the initial position of the dipole and the plate tip, with the $x$ coordinate aligned with the direction of dipole propagation and the $y$ coordinate oriented parallel to the plate in its undeformed state. The plate is thin, that is $h \ll L$, and the deflection is confined to the $x$ direction only and considered small compared to the length of the plate. Consequently, the plate is modeled as a Kirchhoff-Love plate, with density $\rho_{s}$ and bending stiffness per unit width $B$, undergoing pure cylindrical bending ${ }^{1}$. The dipole is completely characterized by its initial advection velocity $U$ and initial radius $a$ (see Peterson and Porfiri (2013) for the pressure and velocity fields associated with a Lamb dipole). The initial spacing between the dipole center and the tip of the plate is selected as $d=4 a$.

The fluid flow is governed by the incompressible Navier-Stokes equations. Selecting the dipole radius and advection speed as the characteristic length and velocity scales, respectively, the Navier-Stokes equations in non-dimensional form are

$$
\begin{gathered}
\nabla \cdot \hat{\mathbf{u}}=0 \\
\frac{\partial \hat{\mathbf{u}}}{\partial \hat{t}}+(\hat{\mathbf{u}} \cdot \nabla) \hat{\mathbf{u}}=-\nabla \hat{P}+\frac{1}{\operatorname{Re}} \nabla^{2} \hat{\mathbf{u}},
\end{gathered}
$$

\footnotetext{
${ }^{1}$ We note that the use of linear plate theory results in an extensible plate. In the current study, which employs a cantilevered plate configuration, the plate tip deflections are less than $0.3 L$. For tip deflections of $0.3 L$, the discrepancy in the tip displacements predicted by linear versus nonlinear theories is expected to be less than $0.05 L$ in the axial direction, and $0.01 \mathrm{~L}$ in the transverse direction, based on the exact solution of an inextensible plate with a follower force at the tip (Timoshenko and Gere, 1961). Given the modest differences we have opted not to complicate the model by adding nonlinearities that do not have an appreciable effect on the attendant fluid-structure interactions.
} 


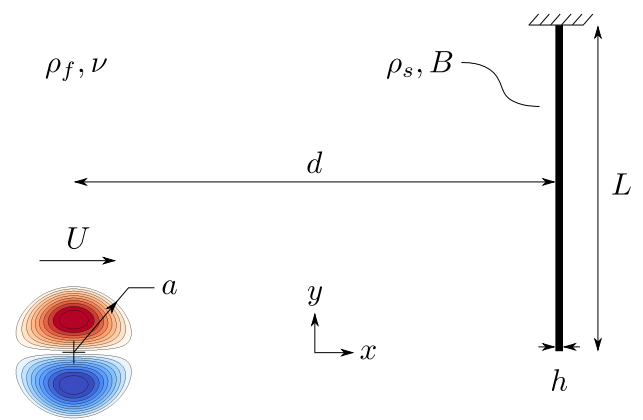

Figure 1: (Color online) Schematic of the problem setup, including pertinent physical parameters and variable definitions.

where $\hat{\mathbf{u}}$ is the non-dimensional velocity, $\hat{P}=P /\left(\rho_{f} U^{2}\right)$ is the non-dimensional pressure, $\operatorname{Re}=U a / \nu$ is the dipole Reynolds number, $\hat{t}=t U / a$ is the nondimensional time coordinate, and $\nabla$ is the $2 \mathrm{D}$ gradient operator with respect to non-dimensional coordinates $\hat{x}$ and $\hat{y}$. The linear-elastic motion of the plate is governed by

$$
\mu \frac{\partial^{2} \hat{\delta}}{\partial \hat{t}^{2}}+K \frac{\partial^{4} \hat{\delta}}{\partial \hat{y}^{4}}=\llbracket \hat{P} \rrbracket(\hat{y}, \hat{t}),
$$

where $\hat{\delta}(\hat{y}, \hat{t})$ is the deflection of the plate centerline in the $x$ direction (scaled with respect to the dipole radius), $\llbracket \hat{P} \rrbracket(\hat{y}, \hat{t})$ is the difference in non-dimensional pressure between the front face (the surface facing the oncoming vortex dipole) and back face of the plate, $\mu=\rho_{s} h /\left(\rho_{f} a\right)$ is the ratio of the characteristic mass of the plate to that of the dipole, and $K=B /\left(\rho_{f} U^{2} a^{3}\right)$ is the non-dimensional bending stiffness, which relates the elastic restoring force in the plate to the applied fluid loading. Note that preliminary results not presented here have shown that viscous stresses on the plate are on the order of $1 \%$ of the applied pressure, and therefore only loading due to pressure in considered. The boundary conditions for the cantilevered plate are

$$
\hat{\delta}(\hat{L}, \hat{t})=\left.\frac{\partial \hat{\delta}}{\partial \hat{t}}\right|_{\hat{L}, \hat{t}}=\left.\frac{\partial^{2} \hat{\delta}}{\partial \hat{t}^{2}}\right|_{0, \hat{t}}=\left.\frac{\partial^{3} \hat{\delta}}{\partial \hat{t}^{3}}\right|_{0, \hat{t}}=0,
$$

where $\hat{L}=L / a$. As posited by Kirchoff-Love plate theory, a given point along the centerline of the plate moves only in the $x$ direction. The cross section of the plate is assumed to remain orthogonal to the centerline and the thickness of the cross section is set to $h=0.08 a$. Note that the plate is extensible, following these assumptions. 
The dimensionless parameters that influence the dynamics of the problem are Re, $\hat{L}, \mu$, and $K$. Simulations are performed at Reynolds numbers of 500,1500 , and 3000, keeping all other parameters fixed at $\hat{L}=4, K=10$, and $\mu=0.016$. These Reynolds numbers represent the range of typical values found in centimeter-scale energy harvesting devices and correspond to the semi-infinite rigid plate simulations presented in Peterson and Porfiri (2013), while the values of $K$ and $\mu$ are based upon the electro-active polymer material employed in Peterson and Porfiri (2012b). A second set of simulations are performed at the same Reynolds numbers but with a rigid plate, that is, with $K \rightarrow \infty$, to assess the influence of plate elasticity on the vortex dynamics.

\section{Solution Procedure}

The numerical solution procedure uses a strongly coupled, staggered FSI algorithm, in which separate solvers are used for the fluid and structural domains, and information such as fluid pressure and structural displacement are exchanged between solvers. At the beginning of each time step, fluid pressure from the previous time step is used as loading on the plate. The plate deflection is then calculated and under-relaxed. The arbitrary Lagrangian-Eulerian (ALE) mesh of the fluid domain is subsequently deformed to accommodate the moving boundary at the interface with the plate, followed by the solution of the fluid flow. This process is repeated until a prescribed convergence tolerance is met. For the current simulations, the tolerance is set on the deflection of the tip of the plate, imposing that the difference in tip deflection between subsequent iterations cannot exceed $\epsilon=10^{-8}$.

\subsection{Structural Solver}

The structural problem is solved employing an implicit finite difference solver, with Eq. (2) discretized using a second order central difference approximation for the spatial derivative and a second order backward difference approximation for the temporal derivative. The discrete version of Eq. (2) is

$$
\mu \frac{2 \delta_{i, k}-5 \delta_{i, k-1}+4 \delta_{i, k-2}-\delta_{i, k-3}}{\Delta t^{2}}+K \frac{\delta_{i+2, k}-4 \delta_{i+1, k}+6 \delta_{i, k}-4 \delta_{i-1, k}+\delta_{i-2, k}}{\Delta y^{4}}=\llbracket P \rrbracket_{i, k},
$$

where the subscript $i$ denotes the discrete point along the plate, and the subscript $k$ denotes the discrete point in time. The use of the "hat" notation 
for non-dimensional variables is dropped for convenience; in this section, all variables are assumed dimensionless unless otherwise noted. It should be noted that for the parameters under consideration in the present study, the first term on the left-hand side of Eq. (4) (inertial term) is three orders of magnitude lower than the second term (elastic stiffness term). The beam is discretized into 40 equally spaced elements of length $\Delta y=0.1$ and the time step is the same as that used in the fluid solver (see Section 3.2). Plate boundary conditions in Eq. (3) are discretized using second order forward and backward difference approximations for the free end and fixed end, respectively. Note that the spectral stability criterion (Ryaben'kii and Tsynkov, 2006) for this finite difference scheme is satisfied for all $K>0$ and $\mu>0$.

As is common in FSI problems, the staggered approach is prone to divergence, and requires under-relaxation of the structural deflection (Kuttler and Wall, 2008). The amount of relaxation and, therefore, the number of subiterations per time step is known to increase with mass ratio (Badia et al., 2008). Here, Aitken's adaptive relaxation (Kuttler and Wall, 2008) is used to accelerate convergence. The iteration number is denoted with a superscript $m$, and the relaxation parameter in the $m^{\text {th }}$ iteration and the $k^{\text {th }}$ time step is denoted $\alpha_{k}^{m}$. The deflection after relaxation is denoted by a tilde as

$$
\tilde{\delta}_{i, k}^{m}=\alpha_{k}^{m} \delta_{i, k}^{m}+\left(1-\alpha_{k}^{m}\right) \tilde{\delta}_{i, k}^{m-1} .
$$

Aitken's relaxation parameter is calculated as

$$
\alpha_{k}^{m}=\alpha_{k}^{m-1} \frac{\sum_{i}\left(r_{i, k}^{m-1}-r_{i, k}^{m}\right) r_{i, k}^{m-1}}{\sum_{i}\left(r_{i, k}^{m-1}-r_{i, k}^{m}\right)^{2}},
$$

where $r_{i, k}^{m}=\delta_{i, k}^{m}-\tilde{\delta}_{i, k}^{m-1}$.

As the plate deforms, the encompassing fluid domain must deform in conjunction. The displacement of the fluid domain is calculated using a Laplacian equation with inverse-square-distance diffusivity (OpenFOAM, 2014). Specifically, the displacement at the interface with the plate is prescribed, and a node of the fluid domain originally at position $(x, y)$ is displaced a distance $\mathbf{D}(x, y)$ according to

$$
\nabla \cdot\left(\frac{\gamma}{R^{2}(x, y)} \nabla \mathbf{D}\right)=0,
$$


where $R(x, y)$ is the average distance of a node to the plate interface and $\gamma$ is a constant.

\subsection{Fluid Solver}

The fluid dynamics is solved using a merged PISO-SIMPLE algorithm, pimpleDyMFoam, in the open-source CFD package OpenFOAM (OpenFOAM, 2014). A no-slip wall condition is imposed on the moving interface with the plate such that the local fluid velocity is equal to the plate velocity and the wall normal pressure gradient is set equal to zero. We note that for the moving wall the latter condition is in general an approximation due to the local shape change and acceleration of the plate. Inlet/outlet boundary conditions are imposed on the domain boundaries such that the total pressure is zero, $\hat{P}+(1 / 2) \hat{\mathbf{u}} \cdot \hat{\mathbf{u}}=0$, and mass flux in or out of the domain is governed by local conservation of momentum.

The 2D fluid domain consists of an outer portion of coarse structured square elements and an inner portion of fine structured square elements. The outer square region has dimensions $51.2 a$ by $51.2 a$ and the inner square region has dimensions $10.24 a$ by $10.24 a$. The inner domain is sized such that it contains all secondary fluid structures resulting from the initial impact for the duration of the simulations, while the outer domain is sized to mitigate the effects of the domain boundary on the solution. Figure 2 shows a diagram of the meshed fluid domain used in the cases of $\operatorname{Re}=500$ and $\operatorname{Re}=1500$, as well as insets that illustrate the mesh resolution relative to the dipole and the plate thickness.

Previous studies (Orlandi, 1990; Morinishi et al., 1998; Shashank et al., 2010) have noted the existence of numerical viscosity in CFD simulations that use non-energy-conserving numerical schemes, such as the OpenFOAM solver employed herein, which can lead to unrealistic vortex dynamics in cases where numerical viscosity is comparable to the physical fluid viscosity. In the present work, the grid size and the time step are carefully selected so as to ensure that the numerical viscosity has a marginal effect. The numerical viscosity is quantified as follows: a dipole is initialized in a domain consisting of four slip walls and zero physical viscosity. Under these conditions, both the total kinetic energy, $E$, and enstrophy, $Z$, of the domain are ideally conserved, with any changes in these properties attributed to numerical dissipation. The numerical viscosity, $\nu_{\text {num }}(t)$, at any time during the simulation can be 


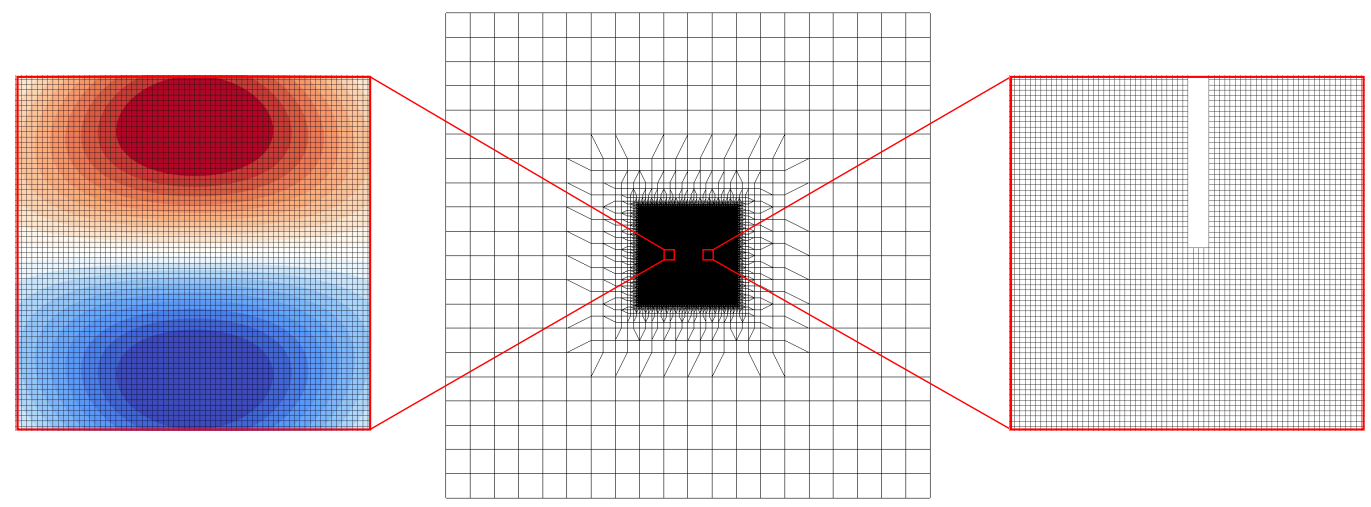

Figure 2: (Color online) Diagram of meshed fluid domain, with detailed views of the plate tip and the initial dipole location.

calculated as (Keetels et al., 2011; Peterson and Porfiri, 2013)

$$
\nu_{\text {num }}(t)=-\frac{\dot{E}(t)}{2 Z(t)},
$$

where the dot denotes differentiation with respect to time. The Reynolds number based on the average numerical viscosity is then defined as $\operatorname{Re}_{\text {num }}=$ $U a / \bar{\nu}_{\text {num }}$, where $\bar{\nu}_{\text {num }}$ is the average of $\nu_{\text {num }}$ over 7.5 time units. See Peterson and Porfiri (2013) for more details on a similar study.

Through this procedures we determined that a time step of $\Delta t=0.001$ and grid spacing of $\Delta x=0.02$ gives a Reynolds number based on average numerical viscosity of $\operatorname{Re}_{\text {num }}=8800$, which is much larger than the physical Reynolds number in the $R e=500$ and $R e=1500$ cases (that is, the numerical viscosity is much lower than the fluid viscosity). The same time step and grid spacing of $\Delta x=0.01$ yield a Reynolds of $R_{\text {num }}=10200$, which is used for the $\mathrm{Re}=3000$ case. Thus, the grid spacing $\Delta x$ for the inner subdomain is selected to be 0.02 for the lower Reynolds number cases and 0.01 for the highest Reynolds number case. A time step of $\Delta t=0.001$ is used throughout all simulations. The grid spacing of the outer subdomain is $\Delta x=2.56$, which is well outside of the region of interest and thus numerical viscosity is not important. Based on the numerical viscosities calculated here, and in the study performed by Peterson and Porfiri (2013), which assesses the numerical viscosity present in a very similar flow simulation, it is determined that the 
time-step size and grid size selected will result in a solution that converges to within the required accuracy.

\subsection{Model Validation}

The validity of the current simulations is ascertained via comparison of rigid plate simulations to published numerical results by Peterson and Porfiri (2013). Although their study observes the impact of a dipole with a rigid, semi-infinite plate, compared to the finite length plate herein, this comparison offers a reasonable first assessment of the accuracy of the proposed approach. For all three Reynolds numbers under investigation, $R e=500,1500$ and 3000, the impact times of the upper secondary dipoles are within $5 \%$ of each other, and the maximum pressure due to these secondary impacts are within $7 \%^{2}$. Additionally, the simulations herein show good qualitative agreement with the numerical results of Orlandi (1990), Clercx and Bruneau (2006), and Kramer et al. (2007), all of whom performed simulations on the impact of a dipole with an infinite wall. Similar features of the vortex dynamics are present in these studies and the work herein, including the rebound effect, and the production of similar tertiary structures.

\section{Results and Discussion}

\subsection{Vortex Dynamics}

As a first point of comparison, the difference in vortex dynamics between the impact of a dipole with the tip of a deformable versus rigid plate is analyzed. Figure 3 shows the evolution of the vorticity field, $\hat{\omega}=\omega a / U$, in both the deformable and rigid cases at $R e=500$, while Figures 4 and 5 show analogous comparisons for $\mathrm{Re}=1500$ and $\mathrm{Re}=3000$, respectively.

For $\mathrm{Re}=500$ (Figure 3), both the rigid and deformable plate cases show similar trends in vortex dynamics up to and shortly after the impact of the dipole with the plate. Particularly, as the dipole approaches the plate $(\hat{t}<4.0)$ positive vorticity is produced at the tip of the plate, and negative vorticity is generated at the front face. At time $\hat{t}=4.0$ the dipole is seen impacting the plate, at which point it splits into two, with each dipole half combining with generated surface vorticity of opposite sign. This produces

\footnotetext{
${ }^{2}$ Note that the time scale used by Peterson and Porfiri (2013) is 6.67 times the time scale used herein, and the dipole is initialized $1 a$ farther from the plate.
} 


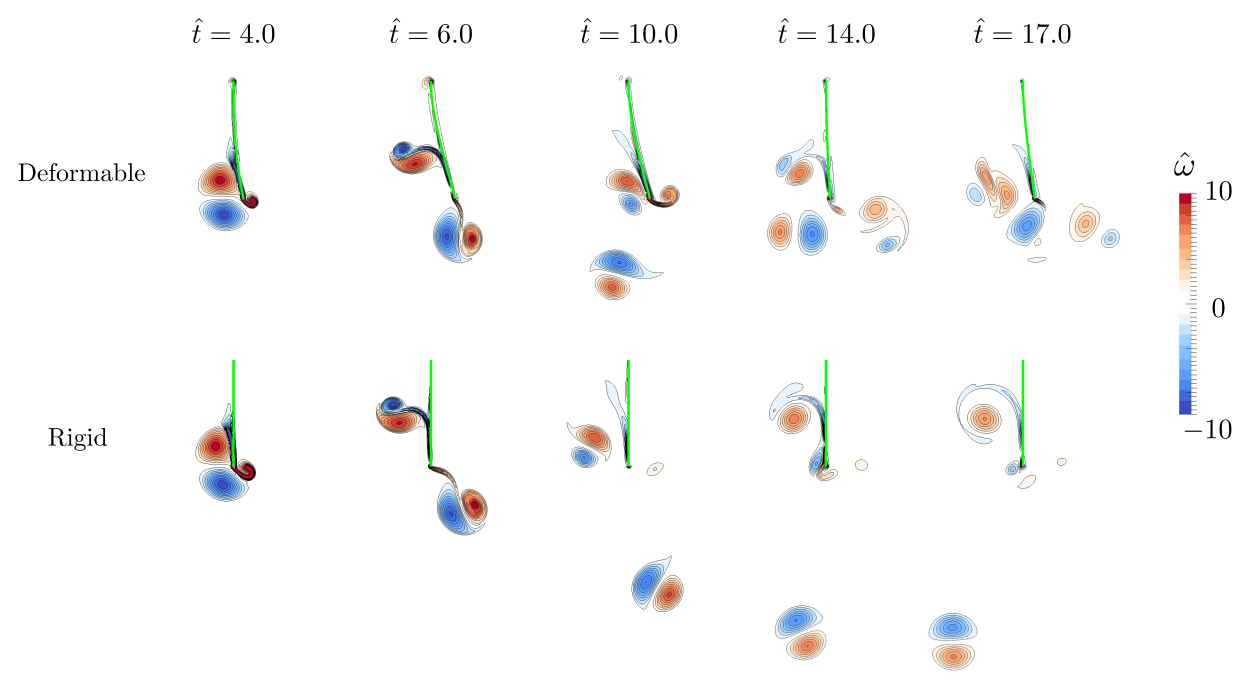

Figure 3: (Color online) Evolution of the vorticity field during impact of a dipole with a deformable (top row) and a rigid (bottom row) plate at $R e=500$. The green line indicates the plate position at each time.

two secondary dipoles, as seen at $\hat{t}=6.0$, referred to as the upper and lower secondary dipoles. The two secondary dipoles propagate with circular trajectories due to their non-zero net circulations. In both the deformable and rigid cases, the upper secondary dipole returns to impact the plate, as seen at $\hat{t}=10.0$, in what is referred to herein as the secondary impact. We comment that the upper secondary dipole impacts the deformable plate sooner than in the rigid case, indicating it has a higher net circulation and, consequently, its trajectory has a smaller radius of curvature. This secondary impact leads to the generation of tertiary vortical structures, as seen at $\hat{t}=$ 14.0.

The difference in vorticity generation at the plate during primary impact between the deformable and rigid cases is also evident when observing the lower secondary dipoles between $\hat{t}=6.0$ and 17.0. As with the upper secondary dipole, the lower secondary dipole follows a trajectory with a smaller radius of curvature in the deformable case, resulting in this dipole returning to impact the plate at $\hat{t}=17.0$, referred to herein as the tertiary impact. In the rigid case, the turning radius of the lower secondary dipole is considerably larger. This dipole advects away from the plate and does not return to impact the plate for the duration of the time frame under investigation. 


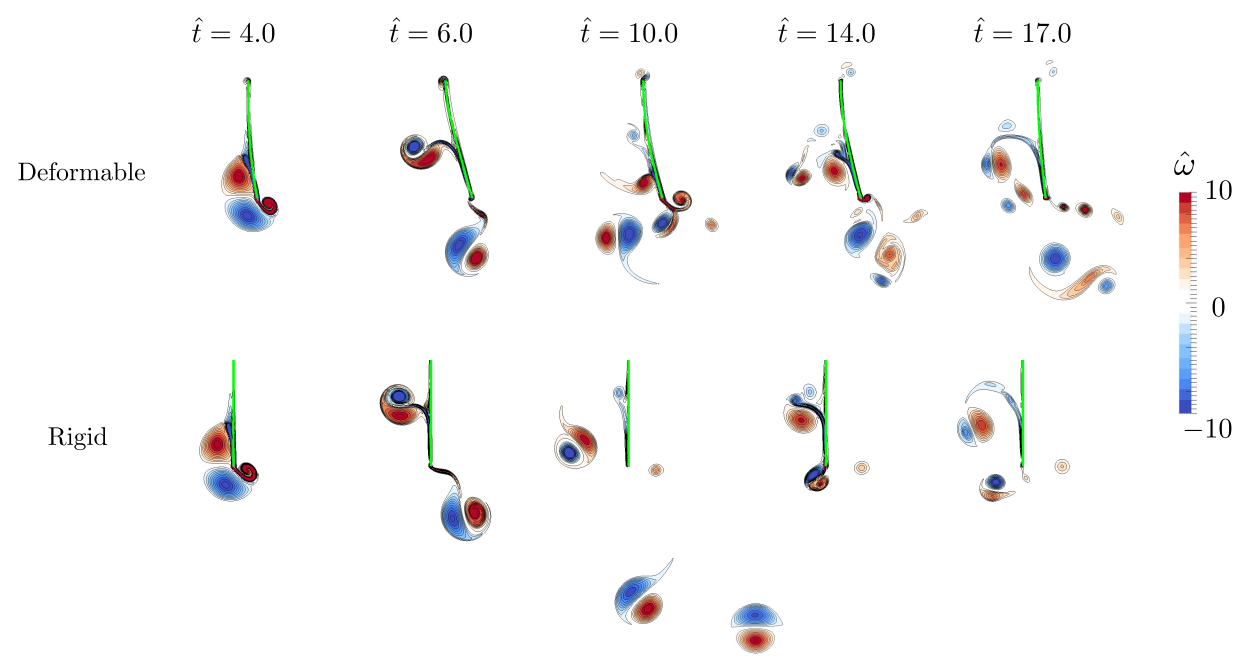

Figure 4: (Color online) Evolution of the vorticity field during impact of a dipole with a deformable (top row) and a rigid (bottom row) plate at $R e=1500$. The green line indicates the plate position at each time.

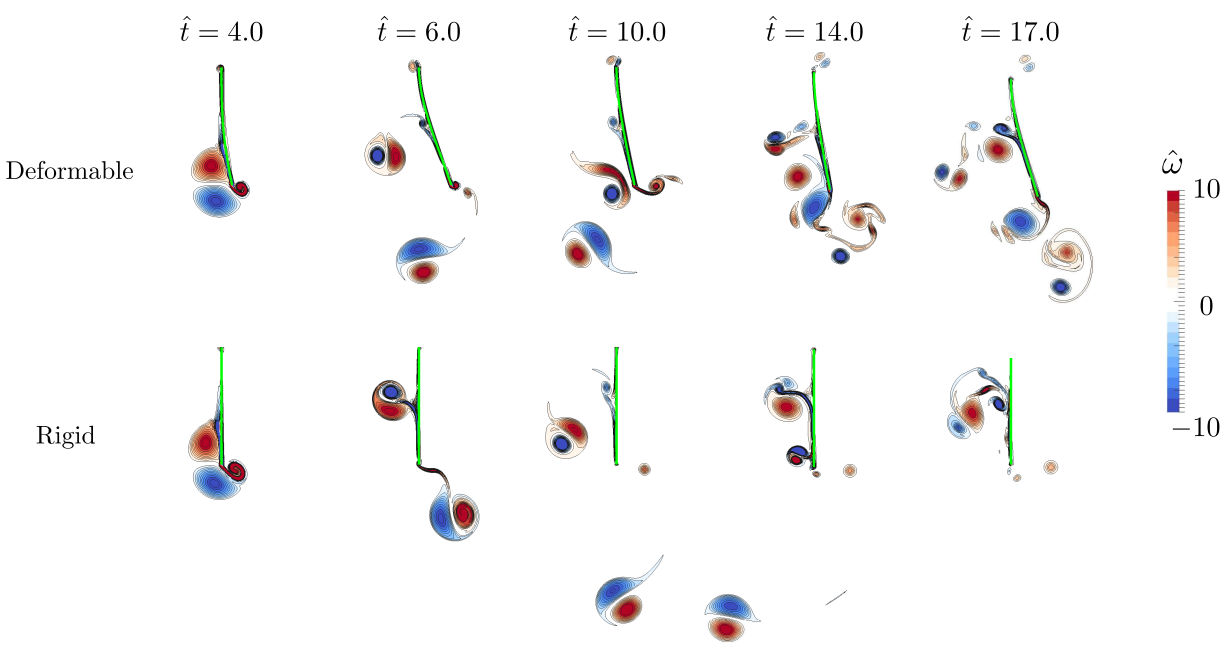

Figure 5: (Color online) Evolution of the vorticity field during impact of a dipole with a deformable (top row) and a rigid (bottom row) plate at $\mathrm{Re}=3000$. The green line indicates the plate position at each time. 
The cases of $\operatorname{Re}=1500$ and $\operatorname{Re}=3000$, presented in Figures 4 and 5 , respectively, show similar vortex dynamics to that observed at $\mathrm{Re}=500$ in the early stages of the impact. Comparing the deformable plate impact at different Reynolds numbers, we find that in all three cases the primary dipole splits in half at $\hat{t}=4.0$ and forms two secondary dipoles, visible at $\hat{t}=6.0$. These secondary dipoles return and generate subsequent secondary and tertiary impacts with the plate, firstly from the upper secondary dipoles at approximately $\hat{t}=10.0$, then from the lower secondary dipoles at $\hat{t}=12.0$ in the case of $\operatorname{Re}=1500$ and $\hat{t}=14.0$ in the case of $\operatorname{Re}=3000$. The effect of Reynolds number on the vortex dynamics becomes prominent after the second and third collisions, with higher Reynolds numbers leading to a larger number of finer tertiary structures.

The reason for this strong Reynolds number dependence after the primary impact is attributed to two factors. The primary factor is vorticity production from no-slip surfaces, which increases with increased Reynolds number. In $2 \mathrm{D}$, the pressure on a no-slip surface is related to vorticity production by (Brons et al., 2014)

$$
-\frac{\partial \hat{\omega}}{\partial \hat{n}}=\operatorname{Re}\left(\frac{\partial \hat{P}}{\partial \hat{s}}+\frac{\mathrm{d} \hat{U}_{s}}{\mathrm{~d} \hat{t}}+\kappa \hat{U}_{n} \hat{U}_{s}-\frac{1}{2} \frac{\partial}{\partial \hat{s}}\left(\hat{U}_{n}^{2}\right)\right),
$$

where $\hat{n}$ and $\hat{s}$ are coordinates normal and tangent to the surface, respectively; $\hat{U}_{n}$ and $\hat{U}_{s}$ are the wall velocities in the $\hat{n}$ and $\hat{s}$ directions, respectively; and $\kappa$ in the local curvature of the surface, non-dimensionalized. The last three terms in Eq. (9) are associated with the movement and deformation of the surface, and in this case are small compared to the other terms, leaving Eq. (9) essentially a balance between vorticity production and the tangential pressure gradient. During the approach of the primary dipole, the pressure gradients induced on the plate are virtually the same at all the Reynolds numbers investigated, resulting in a vorticity flux that is proportional to the Reynolds number. A secondary factor is vorticity diffusion. At higher Reynolds numbers, the rate of vorticity diffusion is reduced, leading to the persistence of finer coherent structures.

Figure 6 displays the ratio of the magnitudes of positive circulation, $\Gamma^{+}$, to the negative circulation, $\Gamma^{-}$, for the two secondary dipoles. For a given dipole, $\Gamma^{+}$is defined as the closed loop line integral of velocity along the contour of constant $\hat{\omega}=1$ and $\Gamma^{-}$is defined as the line integral along the corresponding contour of $\hat{\omega}=-1$. These contour levels are approximately 


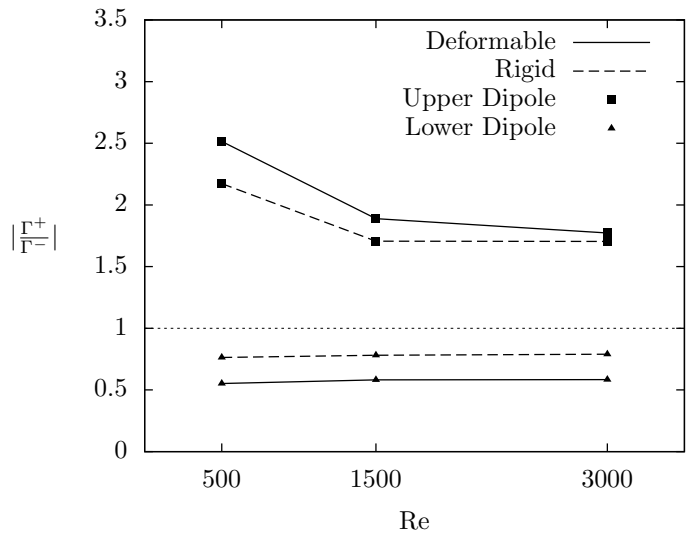

Figure 6: Circulation ratio of secondary dipoles computed when the dipoles separated from the plate (at $\hat{t} \approx 7.0$ ).

$10 \%$ of the maximum/minimum vorticity of the primary dipole. The use of alternate contour levels of $\hat{\omega}= \pm 0.5$ and \pm 2 were found to affect the evaluated circulations by less than $2 \%$. Circulation is evaluated when each dipole separates from the plate (at approximately $\hat{t}=7.0$ in all cases). The moment of separation is defined as the time at which the selected contours, $\hat{\omega}= \pm 1$, detach from the boundary layer vorticity on the plate.

When comparing results from the rigid versus deformable plates, Figure 6 shows that both the upper and lower dipoles consistently have circulation ratios farther from unity in the case of the deformable plate, implying that less vorticity is being produced on this surface. A circulation ratio of unity implies the dipole has a linear trajectory, as both the positive and negative halves of dipole are of equal strength, while a ratio farther from unity implies a smaller radius of curvature. Consequently, secondary dipoles tend to remain closer to the plate in the deformable case, leading to higher pressure loading in the later stages of the interaction. While the circulation ratio of the lower dipole remain virtually independent of Reynolds number, the circulation ratio of the upper dipole decreases with increased Reynolds number. As discussed earlier, this is a consequence of greater vorticity production at the front face of the plate with increased Reynolds number (see Eq. (9)).

\subsection{Plate Loading}

Herein the effects of the plate elasticity and Reynolds number on the hydrodynamic loading on the plate are investigated. Figure 7 presents the 
vorticity field, the pressure distribution on the front face of the deformable plate, and the pressure gradient along the front face at the time of primary dipole impact for $\operatorname{Re}=3000$. Note that the $\hat{y}$ coordinate is measured along

$\hat{P}$

$\begin{array}{lllllllll}2.5 & 2 & 1.5 & 1 & 0.5 & 0 & -0.5 & -1 & -1.5\end{array}$

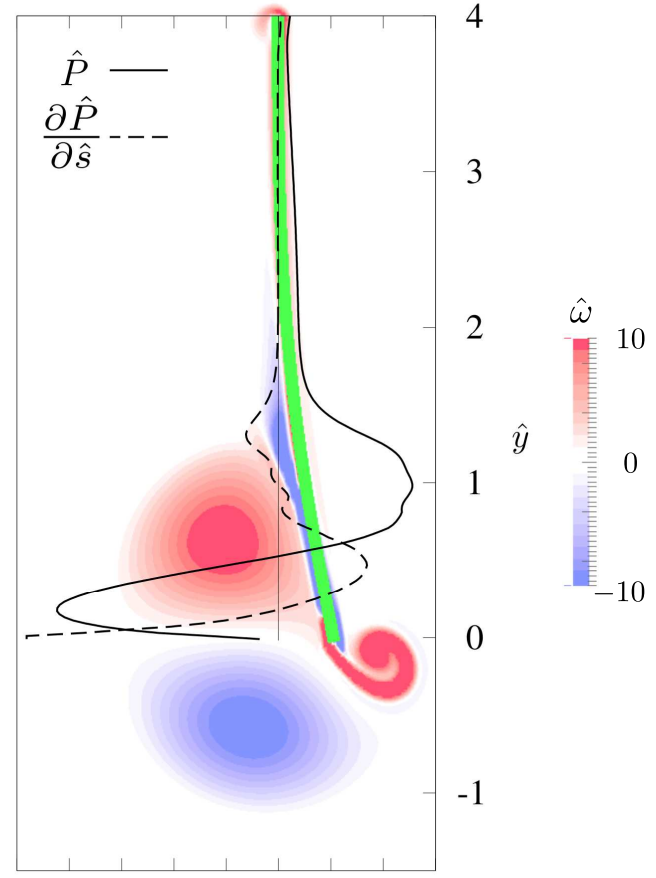

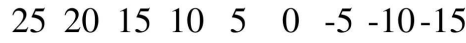

$\frac{\partial \hat{P}}{\partial \hat{s}}$

Figure 7: (Color online) Pressure distribution and pressure gradient on the front face of plate with an overlaid vorticity contour plot at $\hat{t}=4.0$ for $\operatorname{Re}=3000$. The green line indicates the plate location.

the undeformed plate, with $\hat{y}=0$ corresponding to the tip. Similarly, $\hat{s}$ is measured along the deformed plate, with $\hat{s}=0$ at the tip. As the dipole impacts the plate, the greatest pressure is attained very near the tip. This point on the plate also marks a stagnation point, as evident by the two boundary layers which originate at this location. Further up the plate, pressure becomes negative as a result of the proximity of the positive half of the dipole. Three distinct regions of vorticity production are present, corresponding to regions of either positive or negative pressure gradients, consistent with Eq. 
(9). At the tip of the plate, from $0<\hat{y}<0.2$, there is a steep positive pressure gradient, leading to the production of concentrated positive vorticity. This positive vorticity sheds from the tip of the plate, and will ultimately constitute the positive half of the lower secondary dipole. From $0.2<\hat{y}<1$, the negative pressure gradient corresponds to the productions of negative vorticity, which will form half of the upper secondary dipole. Finally, a third region, $1<\hat{y}<4$, is characterized by a relatively small positive pressure gradient, and is associated with minor production of positive vorticity in the corresponding region.

Figures $8(\mathrm{a})$ and $8(\mathrm{~b})$ display the pressure distribution on the front face of the plate, $\hat{P}^{+}$, at several instances in time for the deformable and rigid plates, respectively, at $\operatorname{Re}=500$. At $\hat{t}=4.0$, which corresponds to the impact of

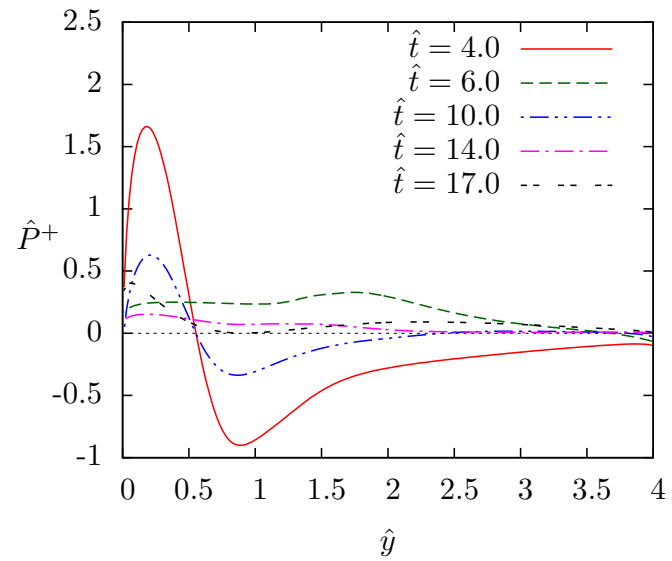

(a)

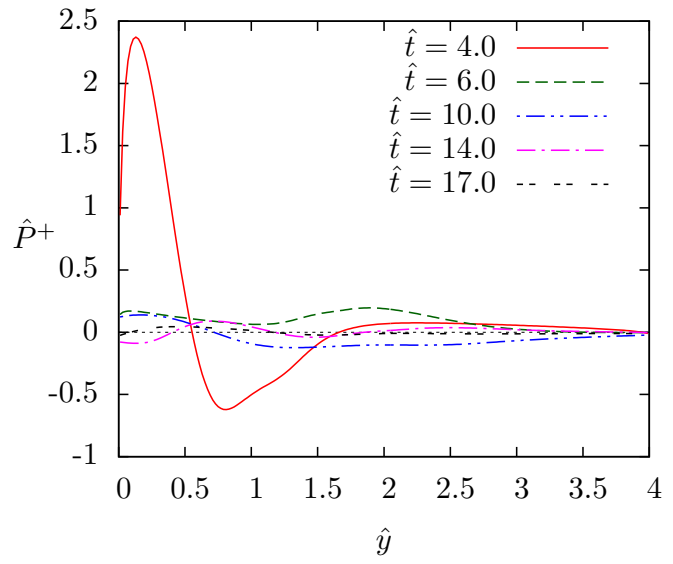

(b)

Figure 8: Pressure loading on the front face of the (a) deformable and (b) rigid plate at $\operatorname{Re}=500$.

the primary dipole, there is a notable difference in the loading between the deformable and rigid cases. In both cases, a large peak is observed near the tip of the plate, however, the peak magnitude is $30 \%$ smaller in the deformable case due to the translation of the plate through the fluid. During impact, the plate deforms in the positive $x$ direction (to the right), causing lower pressure on the front (left) face of the plate and correspondingly higher pressure on the back face.

As was established earlier, weaker vorticity shedding occurs in the deformable plate case. This is corroborated and further elucidated here, as it 
can be seen that this decrease in vorticity production is due to smaller pressure gradients attendant along the surface in the deformable case. The effect of this relative motion of the plate can be observed not only in the diminished pressure maximum at $\hat{t}=4.0$, but also in the large negative value of $\hat{P}^{+}$seen on the deformable plate for $\hat{y}>0.5$. At $\hat{t}=6.0$ and 10.0, the deformable and rigid cases show similar trends, but with greater magnitudes of $\hat{P}^{+}$in the deformable case, again due to the movement of the plate through the fluid. In addition, as a general trend, vortical structures tend to remain closer to the plate in the deformable case, which also contributes to the greater magnitudes of $\hat{P}^{+}$. At the higher Reynolds numbers of 1500 and 3000, similar trends are found, where the peak magnitudes of pressure on the rigid plate are larger during first impact, while peak magnitudes on the deformable plate increase in the later stages of the interaction.

Figure 9 shows the pressure difference between the front and back surfaces of the plate, $\llbracket \hat{P} \rrbracket$, versus $y$-position along the plate at $\hat{t}=4.0$, corresponding to the initial dipole impact, for all three Reynold numbers. Figure 9(a) refers to the case of a deformable plate, and Figure $9(\mathrm{~b})$ pertains to a rigid plate.

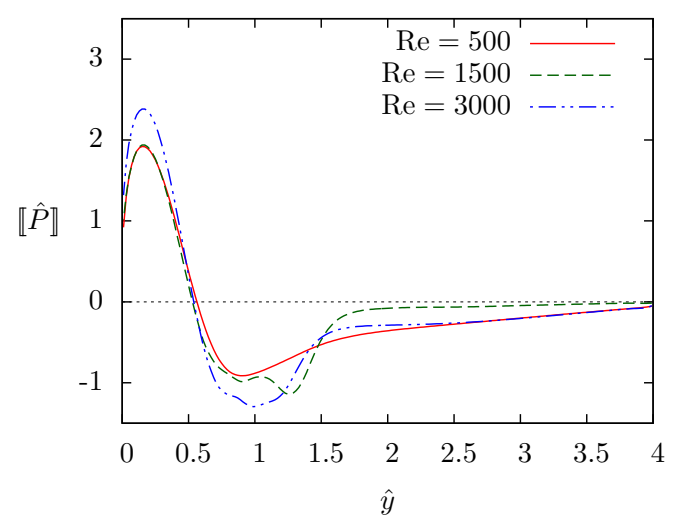

(a)

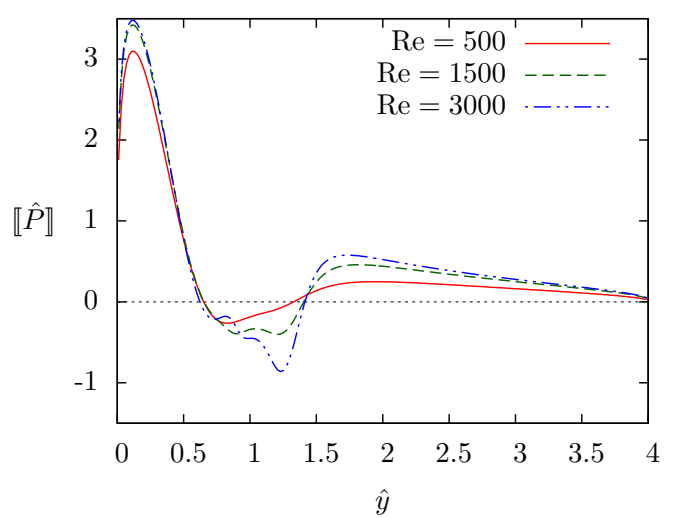

(b)

Figure 9: Pressure difference on a (a) deformable and (b) rigid plate at $\hat{t}=4.0$ for $\operatorname{Re}=$ 500,1500 and 3000 .

Upon impact, the pressure loading varies slightly with Reynolds number, with the general trend that the magnitude of both high and low regions increases with increasing Reynolds number. The loading at initial impact is heavily dependent on the elasticity of the plate, as evidenced by the difference in pressure peak height at the tip between Figures 9(a) and 9(b), while 
depending less on Reynolds number. However, Reynolds number does play a significant role on the post-impact vortex dynamics, and therefore on the loading at later times.

\subsection{Deformation}

Figures 10, 11 and 12 show plate tip deflection, $\hat{\delta}_{\text {tip }}$, versus time for Re $=500,1500$ and 3000, respectively. To relate the plate response to relevant vortex dynamics, insets of the vorticity field are added at select times. Throughout the interaction, the plate deflection is primarily along its fundamental mode shape, therefore the tip deflection is sufficient to describe the plate kinematics.

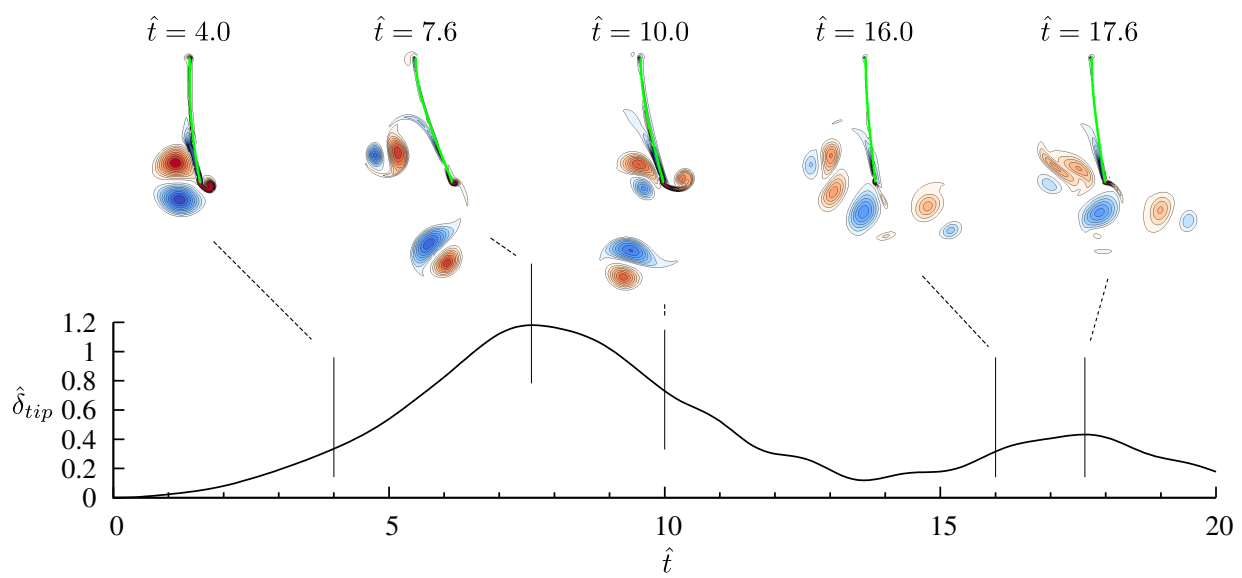

Figure 10: Deflection of plate tip versus time at $R e=500$. The corresponding vorticity field is presented in insets at times corresponding to the initial dipole impact $(\hat{t}=4.0)$, peak tip deflection $(\hat{t}=7.6)$, secondary impact $(\hat{t}=10.0)$, tertiary impact $(\hat{t}=16.0)$, and the second tip deflection peak $(\hat{t}=17.6)$.

Examining first the case of $\operatorname{Re}=500$ (Figure 10), the plate begins to deflect prior to the impact of the primary dipole due to the stagnation pressure on the plate caused by the approaching dipole. This is also found in the potential flow model of Peterson and Porfiri (2012a), in which the plate deflection slowly increases as a vortex pair approaches. Unlike the potential flow model, the displacement of the plate continues to increase after impact at $\hat{t}=4.0$ until a maximum deflection is reached at $\hat{t}=7.6$. After $\hat{t}=7.6$, the plate begins to return to its equilibrium position. During this phase, the plate is impacted by the upper secondary dipole at $\hat{t}=10.0$. However, 


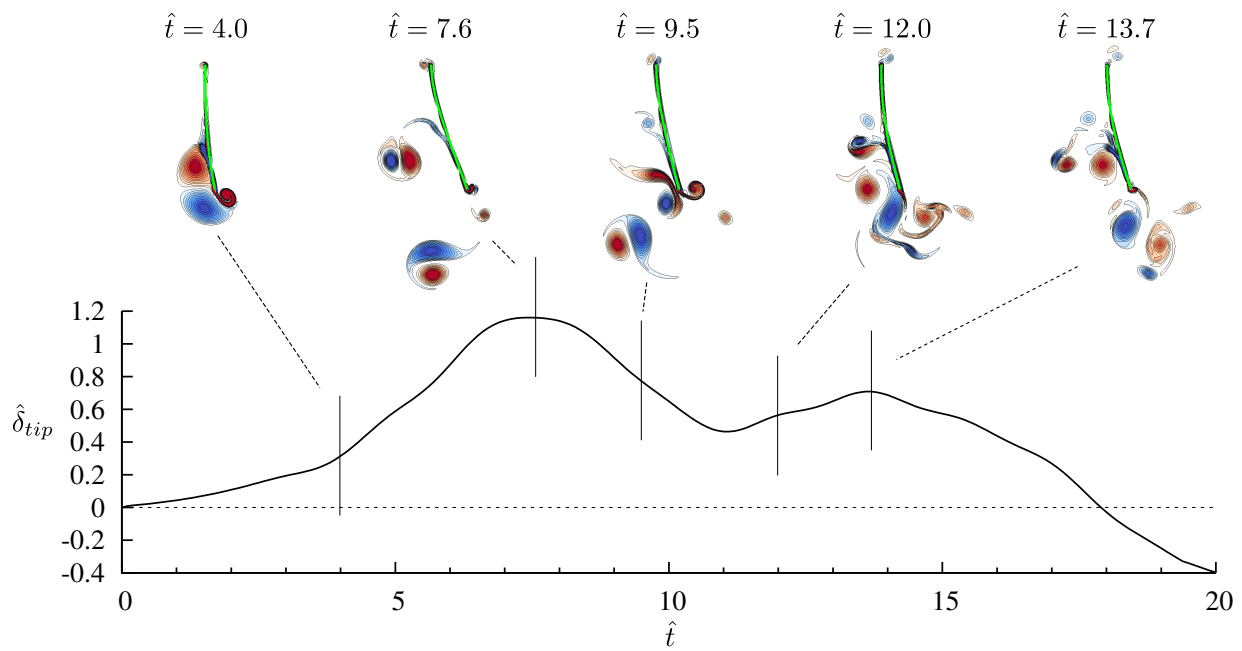

Figure 11: Deflection of plate tip versus time at $R e=1500$. The corresponding vorticity field is presented in insets at times corresponding to the initial dipole impact $(\hat{t}=4.0)$, peak tip deflection $(\hat{t}=7.6)$, secondary impact $(\hat{t}=10.0)$, tertiary impact $(\hat{t}=16.0)$, and the second tip deflection peak $(\hat{t}=17.6)$.

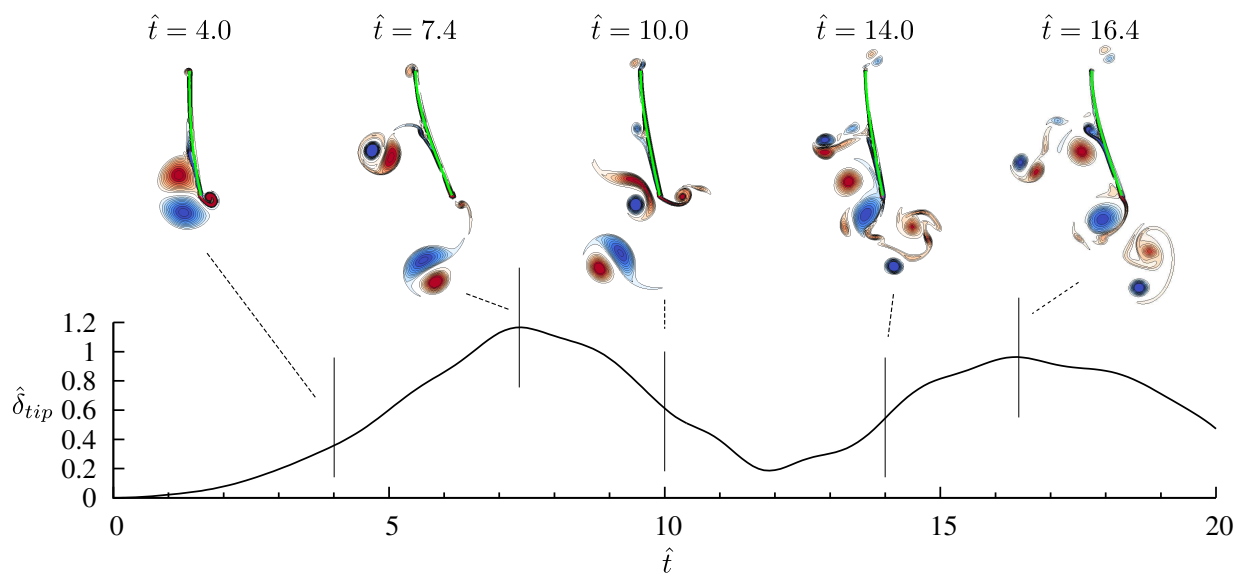

Figure 12: Deflection of plate tip versus time at $R e=3000$. The corresponding vorticity field is presented in insets at times corresponding to the initial dipole impact $(\hat{t}=4.0)$, peak tip deflection $(\hat{t}=7.6)$, secondary impact $(\hat{t}=10.0)$, tertiary impact $(\hat{t}=16.0)$, and the second tip deflection peak $(\hat{t}=17.6)$. 
this collision produces no significant changes in tip deflection. At approximately $\hat{t}=13.5$, the plate again begins to deflect in the positive $x$-direction, presumably due to the increased stagnation pressure on the plate due to the approach of the lower secondary dipole. At $\hat{t}=16.0$, the plate is impacted by the lower secondary dipole as it moves in the positive $x$-direction. This impact continues to force the plate to deflect in the positive $x$ direction, which produces a second peak in deflection at $\hat{t}=17.6$. We note that the surrounding fluid has the effect of overdamping the vibration of the plate. The plate does not oscillate at its in vacuum natural frequency (the time scale of which is much shorter than the convective time scale used throughout), but instead slowly returns to its equilibrium position upon being perturbed. Structural damping is therefore shown to be a secondary phenomenon compared to the external fluid (viscous) damping, and the neglecting of structural damping in the current model is justified.

The higher Reynolds number cases (Figures 11 and 12) show similar trends to those seen at $\operatorname{Re}=500$. There is a large peak in tip deflection in response to the primary impact, the magnitude of which shows very little Reynolds number dependence. As in the $R e=500$ case, there are two subsequent impacts, first from the upper, then the lower secondary dipoles, and a second peak in tip deflection due to the action of the secondary dipoles. It can be seen in the vorticity contour plots in Figures 10, 11 and 12 that the vorticity of secondary structures is higher at larger Reynolds number, due to reduced viscous diffusion. Therefore, the pressure field induced by these structures increases with Reynolds number, leading to a greater second peak in tip deflection. Note that the time of the second peak changes considerably with Reynolds number in a non-monotonic manner; the second peak occurs sooner for the $\mathrm{Re}=1500$ case in comparison with $\mathrm{Re}=500$ and 3000. This is likely associated with the difference in the secondary dipole impact times

with Reynolds number, occurring at $\hat{t}=16.0, \hat{t}=12.0$, and $\hat{t}=14.0$ for the $\mathrm{Re}=500,1500$, and 3000 cases, respectively.

\subsection{Energy}

The initial kinetic energy of the Lamb dipole per unit width, $E_{0}$, can be obtained by integration over the infinite fluid domain (Peterson and Porfiri, 2013) as

$$
E_{0}=\frac{1}{2} \int_{-\infty}^{\infty} \int_{-\infty}^{\infty} \mathbf{u} \cdot \mathbf{u} \mathrm{d} x \mathrm{~d} y=2 \pi \rho_{f} U^{2} a^{2}
$$


To elucidate the amount of energy transferred from the dipole to the plate, $E_{0}$ is used to normalize the strain and kinetic energy of the plate, such that the plate energy is expressed as a fraction of the energy of the dipole. The normalized plate strain energy, $\hat{E}_{s}$, and kinetic energy, $\hat{E}_{k}$, are computed as

$$
\begin{gathered}
\hat{E}_{s}=\frac{E_{s}}{E_{0}}=\frac{K}{4 \pi} \int_{0}^{\hat{L}}\left(\frac{\partial^{2} \hat{\delta}}{\partial \hat{y}^{2}}\right)^{2} \mathrm{~d} \hat{y}, \\
\hat{E}_{k}=\frac{E_{k}}{E_{0}}=\frac{\mu}{4 \pi} \int_{0}^{\hat{L}}\left(\frac{\partial \hat{\delta}}{\partial \hat{t}}\right)^{2} \mathrm{~d} \hat{y} .
\end{gathered}
$$

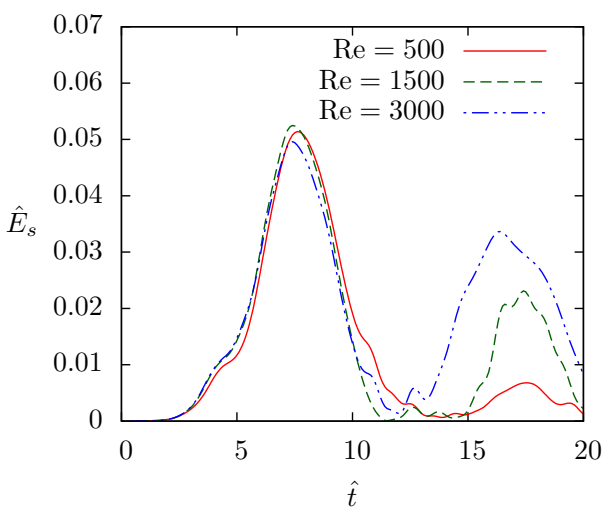

(a)

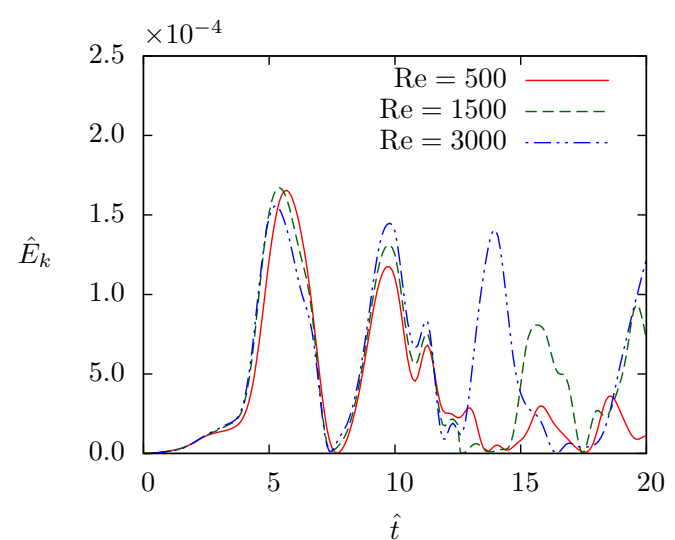

(b)

Figure 13: (a) Strain energy and (b) kinetic energy of the plate versus time for the three Reynolds number cases considered.

Figures 13(a) and 13(b) illustrate the temporal evolution of the strain energy and kinetic energy of the plate, respectively, for the three Reynolds numbers considered. The results demonstrate that the strain energy dominates, exceeding the kinetic energy by approximately two orders of magnitude, which was expected due to the modest value of $\mu$. A comparison of Figure 13(a) with Figures 10 - 12 reveals a strong correlation between the strain energy and tip deflection, corroborating the observation that the plate's response is associated primarily with the fundamental mode. As with tip deflection, the plate energy shows minor Reynolds number dependence for $\hat{t}<10.0$. All three Reynolds number cases show a gradual increase in the strain energy as the primary dipole approaches the plate, followed by a 
rapid increase after impact at $\hat{t}=4.0$. This was also observed experimentally for a vortex ring impacting a deformable electro-active strip in Peterson and Porfiri (2012b). The maximum strain energy is reached at $\hat{t}=7.5$, with the peak value being approximately equal for all cases. The peak value of strain energy is approximately $5 \%$ of the total energy of the original dipole. For all Reynolds numbers investigated, a second maximum in strain energy is achieved due to the impact of the lower secondary dipoles. This maximum is reached between $\hat{t}=16.0$ and $\hat{t}=17.5$, depending on Reynolds number. Due to viscous diffusion, the strength of these secondary dipoles upon collision is larger for higher Reynolds numbers, resulting in a higher second peak in strain energy at increased Reynolds numbers.

Similar to strain energy, the kinetic energy is found to be independent of Reynolds number for $\hat{t}<10.0$. Kinetic energy increases to a maximum value shortly after the primary impact, at $\hat{t}=5.0$. At $\hat{t}=7.5$, the plate momentarily stops (reaches zero kinetic energy and maximum strain energy) and at $\hat{t}=10.0$, a second maximum is attained as the plate returns to its equilibrium position. Multiple smaller peaks are seen at all three Reynolds numbers at $\hat{t}>10.0$, but the timing and magnitude of these peaks varies significantly with Reynolds number.

\section{Conclusions}

The impact of a vortex dipole with the tip of a deformable and rigid plate has been investigated numerically for dipole Reynolds numbers of 500, 1500, and 3000. The study examines the interrelation between the attendant vortex dynamics, plate response, and loading. The results show similar underlying trends in vortex dynamics for all the Reynolds numbers investigated. Specifically, negative and positive vorticity is produced on the front surface and tip of the plate, respectively, as the primary dipole approaches the plate. This surface-generated vorticity then combines with the opposite-sign halves of the primary dipole to form two secondary dipoles.

The produced secondary dipoles have non-zero net circulation, which produces circular dipole trajectories and leads to secondary dipole impacts on the plate at all the Reynolds numbers investigated. However, the rate of production of vorticity at the plate surface increases with increasing Reynolds number, which decreases the net circulation of the secondary dipoles. Consequently, following the initial impact, vortex dynamics show a significant dependence on the Reynolds number. At higher Reynolds numbers, the 
lower net circulation of secondary dipoles cause their trajectories to have larger radii of curvature and cause these dipoles to impact the plate at different times. After the impact of the secondary dipoles, the effect of Reynolds number on vortex dynamics increases further, with more numerous and complex tertiary structures being produced at higher Reynolds number. The increasing complexity of the vortex dynamics is due to both the increasing production rate of vorticity at the surface of the plate and the decreasing viscous dissipation.

Comparison at all three Reynolds numbers with numerical results by Peterson and Porfiri (2013) show that the effect of using a rigid finite plate of $L / a=4$, versus a rigid semi-finite plate $(L / a \rightarrow \infty)$, results in a slight change to the secondary impact times and maximum pressures on the structure. It is expected that smaller values of $L / a$ will have more pronounced differences with the semi-infinite plate results.

The main peak in tip deflection and the associated loading on the plate correspond to the impact of the primary dipole and do not change appreciably within the range of Reynolds numbers examined. A secondary peak in plate deflection is associated with the impact of the lower secondary dipole. Due to the associated changes in vortex dynamics, increasing Reynolds number produces a larger secondary deflection. After the impact of the secondary dipoles, the presence of stronger and more numerous tertiary structures leads to larger unsteady loads on the plate at higher Reynolds number, and therefore more energy transfer during the later stage of the fluid-structure interaction.

The compliance of the plate is found to have a significant effect on vortex dynamics and plate loading. While the rigid plate experiences higher loading during the primary impact, it has a higher rate of vorticity production at the surface compared to the deformable plate. This leads to the formation of secondary dipoles with lower net circulation for the rigid plate, with longer periods between secondary dipole impacts and the overall reduction in loading on the rigid plate subsequent to the primary impact. The results emphasize the strongly coupled nature of this problem, as vortex dynamics and plate deformation each have a significant effect on the other.

In addition to elucidating the vortex dynamics and its effect on plate loading, this study also presents estimates for the energy transfer possible between coherent fluid structures and a deformable plate, which is on interest for the application of energy harvesting. It should be noted, however, that several assumptions incorporated into the present model differ from the conditions 
typically encountered in energy harvesting applications. For instance, while the role of structural damping or an electrical shunt (Fleming et al., 2002) are expected to be small compared to viscous damping, these effects will be present when using an electro-active polymer or another active material as the cantilevered plate. Additionally, coherent structures found in nature, such as vortex rings, are three-dimensional, whereas three-dimensional effects, such as vortex stretching and edge effects (Facci and Porfiri, 2012), have been neglected in the present simulations. It is of interest in future investigations to relate this work back to the impact of a vortex ring with a deformable plate and quantify under what conditions three-dimensional effects become significant.

\section{Acknowledgments}

This work is supported in part by the Natural Sciences and Engineering Research Council in Canada under grant no. 386282, the National Science Foundation under grant no. CBET-1332204 and by the Office of Naval Research under grant no. N00014-10-1-0988, with Dr. Y. D. S. Rajapakse as the program manager. Views expressed herein are those of authors and not of the funding agencies.

\section{References}

Akaydin, H. D., Elvin, N., Andreopoulos, Y., 2010a. Energy harvesting from highly unsteady fluid flows using piezoelectric materials. Journal of Intelligent Material Systems and Structures 21, 1263-1278.

Akaydin, H. D., Elvin, N., Andreopoulos, Y., 2010b. The performance of a self-excited fluidic energy harvester. Experiments in Fluids 49, 291304.

Akaydin, H. D., Elvin, N., Andreopoulos, Y., 2012. The performance of a self-excited fluidic energy harvester. Smart Materials and Structures 21, 025007.

Allen, J. J., Smits, A. J., 2001. Energy harvesting eel. Journal of Fluids and Structures 15, 629-640.

Badia, S., Quaini, A., Quarteroni, A., 2008. Modular vs. non-modular preconditioners for fluidstructure systems with large added-mass effect. Computer Methods in Applied Mechanics and Engineering 197, 4216-4232. 
Brons, M., Thompson, M. C., Leweke, T., Hourigan, K., 2014. Vorticity generation and conservation for two-dimensional interfaces and boundaries. Journal of Fluid Mechanics 758, 63-93.

Cellini, F., Peterson, J. P. S. D., Porfiri, M., 2014. Underwater energy harvesting from a turbine hosting ionic polymer metal composites. Smart Materials and Structures 23, 085023.

Clercx, H. J. H., Bruneau, C. H., 2006. The normal and oblique collision of a dipole with a no-slip boundary. Computers and Fluids 35, 245-279.

De Marqui Jr., C., Erturk, A., 2012. Electroaeroelastic analysis of airfoilbased wind energy harvesting using piezoelectric transduction and electromagnetic induction. Journal of Intelligent Material Systems and Structures 24, 846854 .

Facci, A. L., Porfiri, M., 2012. Nonlinear hydrodynamic damping of sharpedged cantilevers in viscous fluids undergoing multi-harmonic base excitation. Journal of Applied Physics 112, 124908.

Fleming, A. J., Behrens, S., Moheimani, S. O. R., 2002. Optimization and implementation of multimode piezoelectric shunt damping systems. IEEE/ASME Transactions on Mechatronics 7, 87-94.

Giacomello, A., Porfiri, M., 2011. Underwater energy harvesting from a heavy flag hosting ionic polymer metal composites. Journal of Applied Physics 109, 084903.

Goushcha, O., Elvin, N., Andreopoulos, Y., 2014. Interactions of vortices with a flexible beam with applications in fluidic energy harvesting. Applied Physics Letters 104, 021919.

Keetels, G. H., Kramer, W., Clercx, H. J. H., van Heijst, G. J. F., 2011. On the reynolds number scaling of vorticity production at no-slip walls during vortex-wall collisions. Theoretical and Computational Fluid Dynamics 25, 293-300.

Kramer, W., Clercx, H. J. H., van Heijst, G. J. F., 2007. Vorticity dynamics of a dipole colliding with a no-slip wall. Physics of Fluids 19, 126603. 
Kuttler, U., Wall, W. A., 2008. Fixed-point fluid-structure interaction solvers with dynamic relaxation. Computational Mechanics 43, 61-72.

Lamb, H., 1932. Hydrodynamics. Cambridge University Press, Cambridge, U.K.

Lim, T. T., Nickels, T. B., Chong, M. S., 1991. A note on the cause of rebound in the head-on collision of a vortex ring with a wall. Experiments in Fluids 12, 41-48.

Michelin, S., Doare, O., 2013. Energy harvesting efficiency of piezoelectric flags in axial flows. Journal of Fluid Mechanics 714, 489-504.

Milne-Thomson, L. M., 1996. Theoretical Hydrodynamics. Dover Publications Inc., New York, New York.

Morinishi, Y., Lund, T. S., Vasilyev, O. V., Moin, P., 1998. Fully conservative higher order finite difference schemes for incompressible flow. Journal of Computational Physics 143, 90-124.

Myers, R., Vickers, M., Kim, H., 2007. Small scale windmill. Applied Physics Letters 90, 054106.

Nemat-Nasser, S., Li, J. Y., 2000. Electromechanical response of ionic polymer-metal composites. Journal of Applied Physics 87, 3321-3331.

OpenFOAM, 2014. OpenFOAM Users Guide. The OpenFOAM Foundation.

Orlandi, P., 1990. Vortex dipole rebound from a wall. Physics of Fluids A 2, 1429-1436.

Peace, A. J., Riley, N., 1983. A viscous vortex pair in ground effect. Journal of Fluid Mechanics 129, 409-426.

Peterson, S. D., Porfiri, M., 2012a. Energy exchange between a vortex ring and an ionic polymer metal composite. Applied Physics Letters 100, 114102.

Peterson, S. D., Porfiri, M., 2012b. Interaction of a vortex pair with a flexible plate in an ideal quiescent fluid. Journal of Intelligent Material Systems and Structures 23, 14851504. 
Peterson, S. D., Porfiri, M., 2013. Impact of a vortex dipole with a semiinfinite rigid plate. Physics of Fluids 25, 093103.

Priya, S., Chen, C., Fye, D., Zahnd, J., 2005. Piezoelectric windmill: A novel solution to remote sensing. Japanese Journal of Applied Physics 44, 104-107.

Ryaben'kii, V. S., Tsynkov, S. V., 2006. A Theoretical Introduction to Numerical Analysis. CRC Press, Boca Raton, Florida.

Saffman, P. G., 1979. The approach of a vortex pair to a plane surface in inviscid fluid. Journal of Fluid Mechanics 92, 497-503.

Shahinpoor, M., Kim, K. J., 2001. Ionic polymer-metal composites: I. Fundamentals. Smart Materials and Structures 10, 819-833.

Shashank, Larsson, J., Iaccarino, G., 2010. A co-located incompressible navierstokes solver with exact mass, momentum and kinetic energy conservation in the inviscid limit. Journal of Computational Physics 229, 44254430 .

Timoshenko, S. P., Gere, J. M., 1961. Theory of Elastic Stability. McGrawHill Inc., New York, New York.

van Heijst, G., Flor, J., 1989. Laboratory experiments on dipole structures in a stratified fluid. Elsevier Oceanography Series 50, 591-608.

Weinstein, L. A., Cacan, M. R., So, P. M., Wright, P. K., 2012. Vortex shedding induced energy harvesting from piezoelectric materials in heating, ventilation and air conditioning flows. Smart Materials and Structures 21, 045003 . 
$\rho_{f}, \nu$

$\rho_{s}, B$

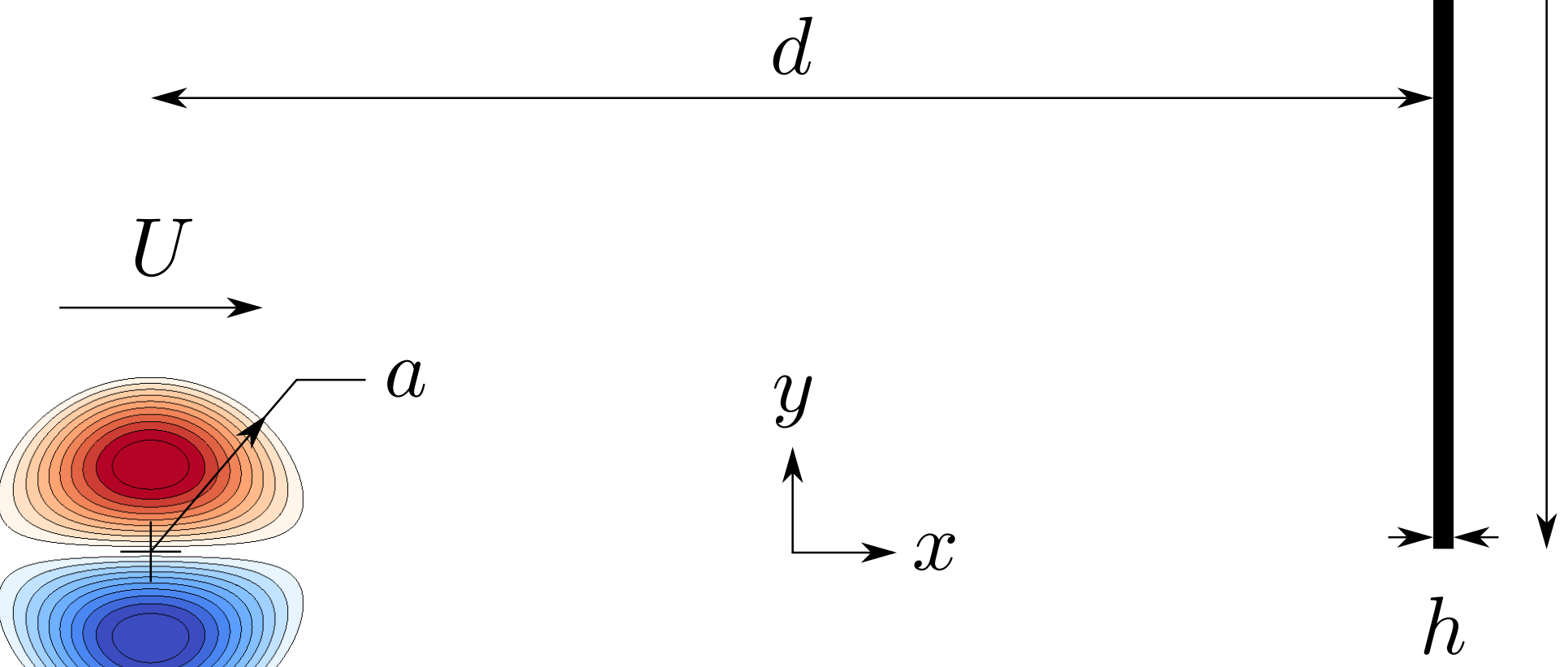

$d$

$y$

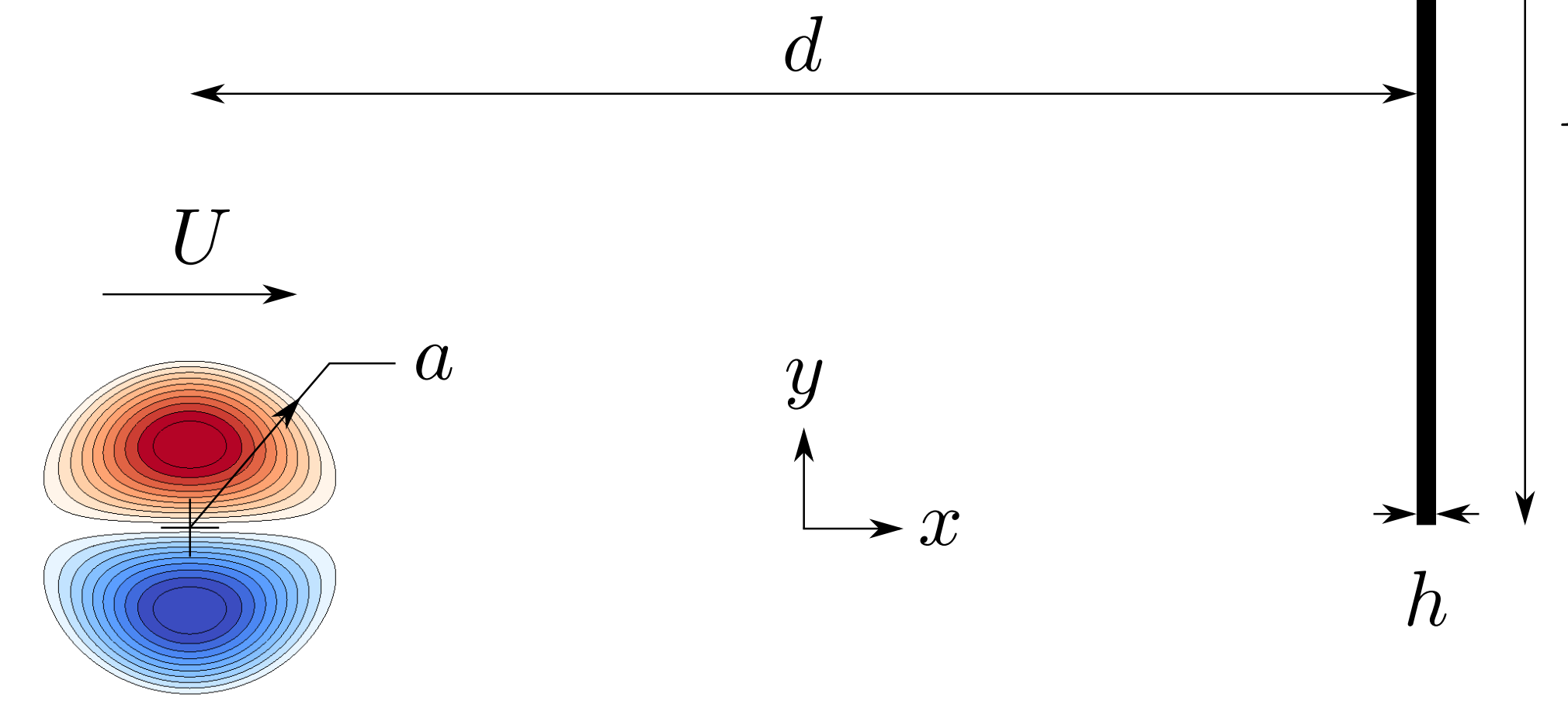

$a$
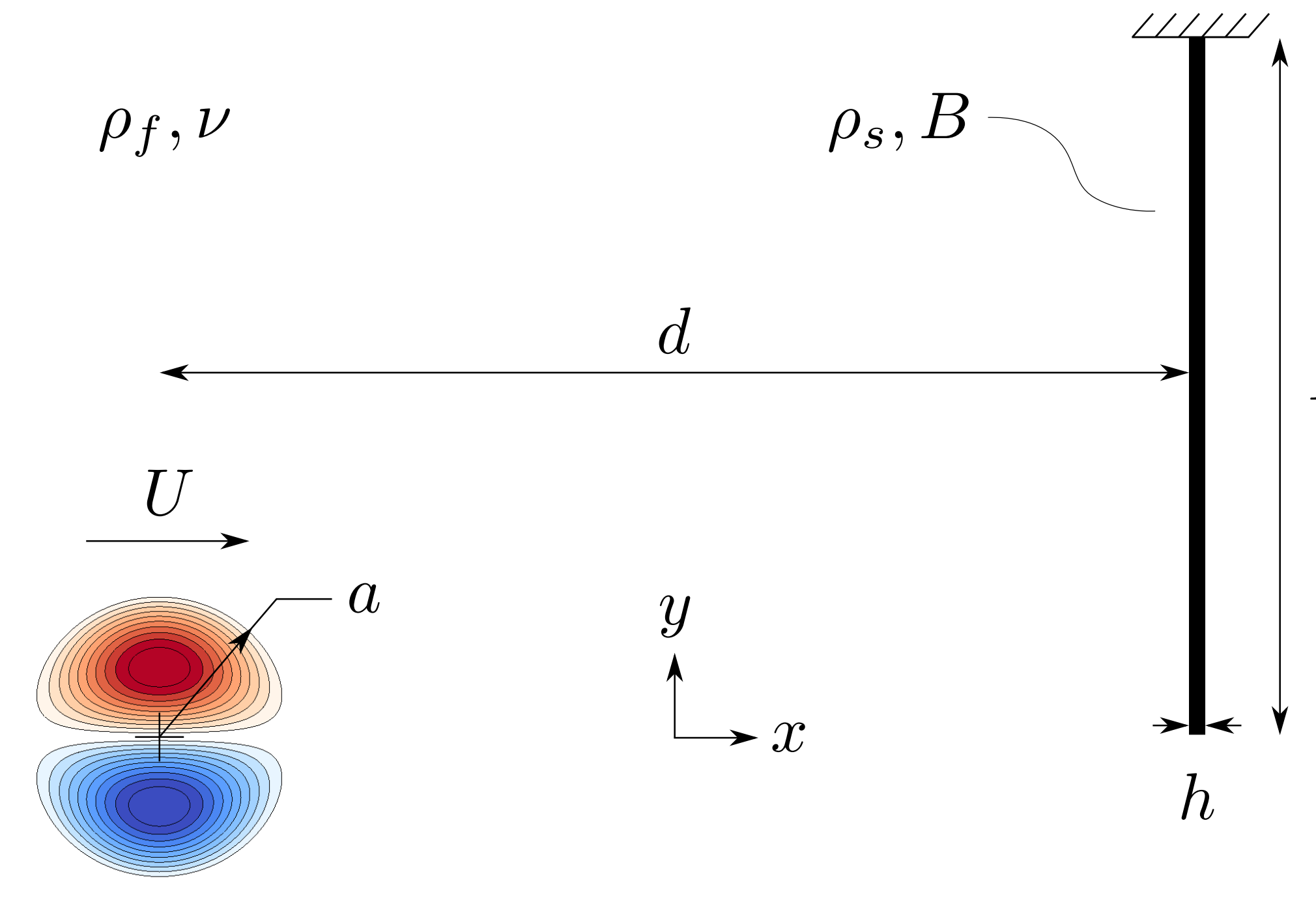

$L$

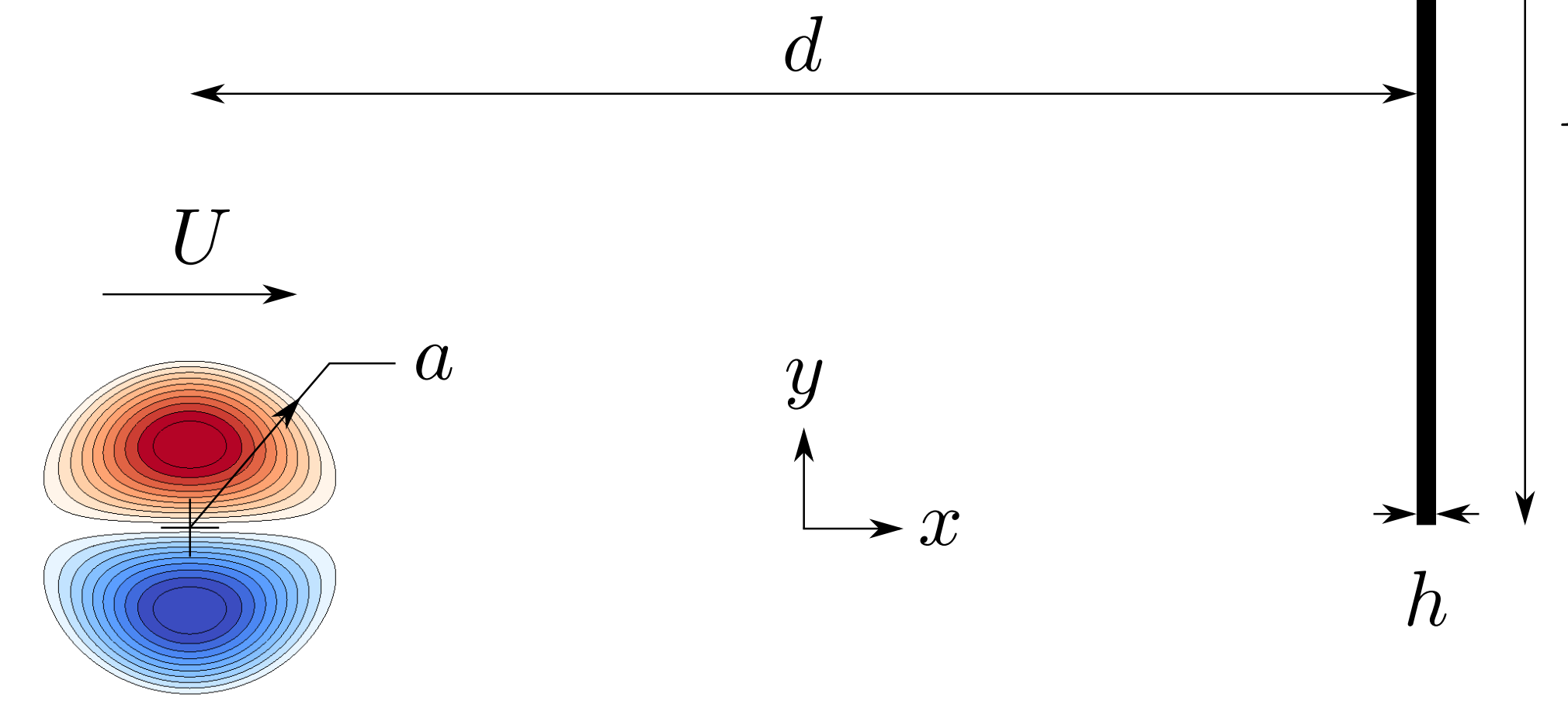

$x$

$\rightarrow \leftarrow \downarrow$

$h$

Figure
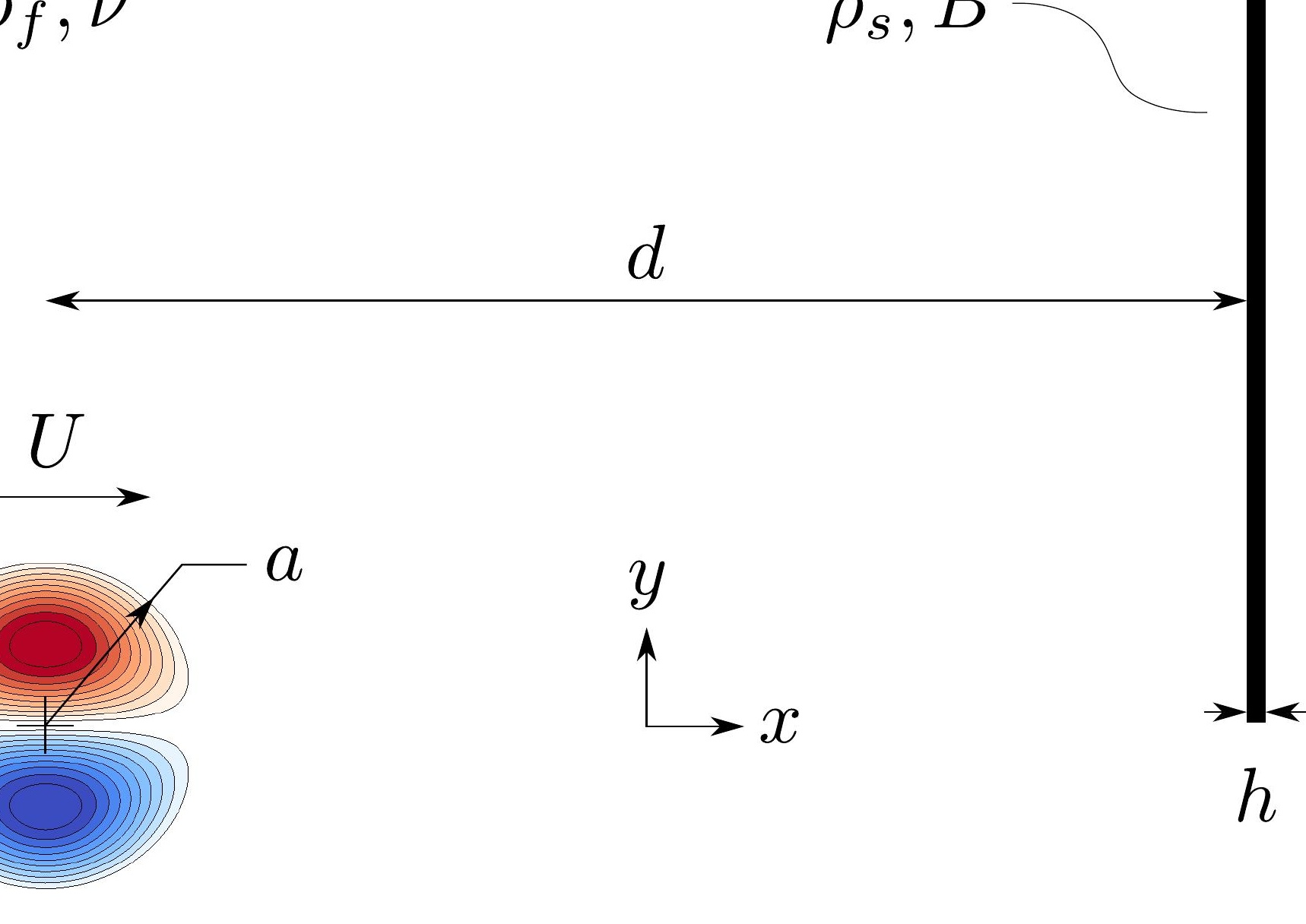


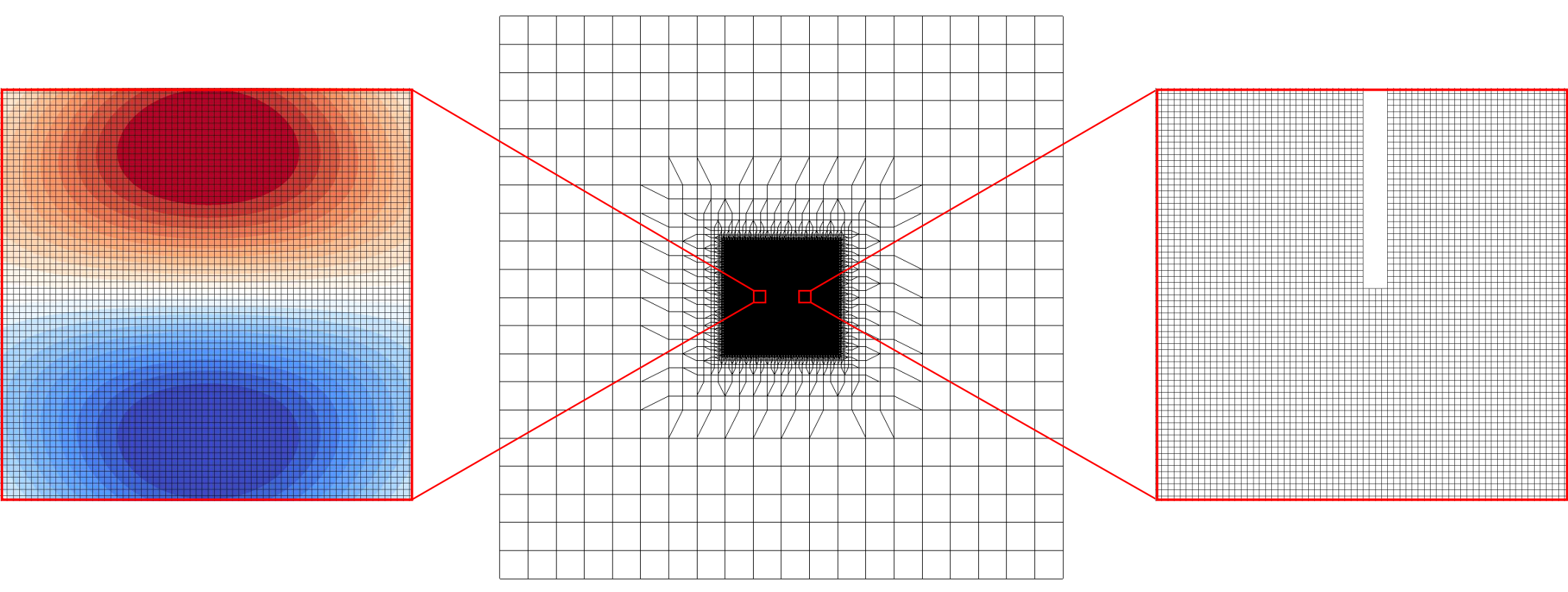

Figure

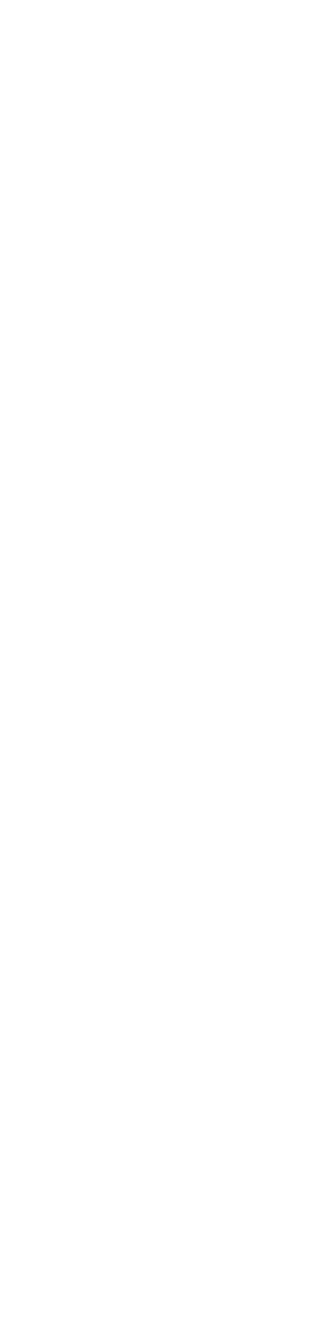



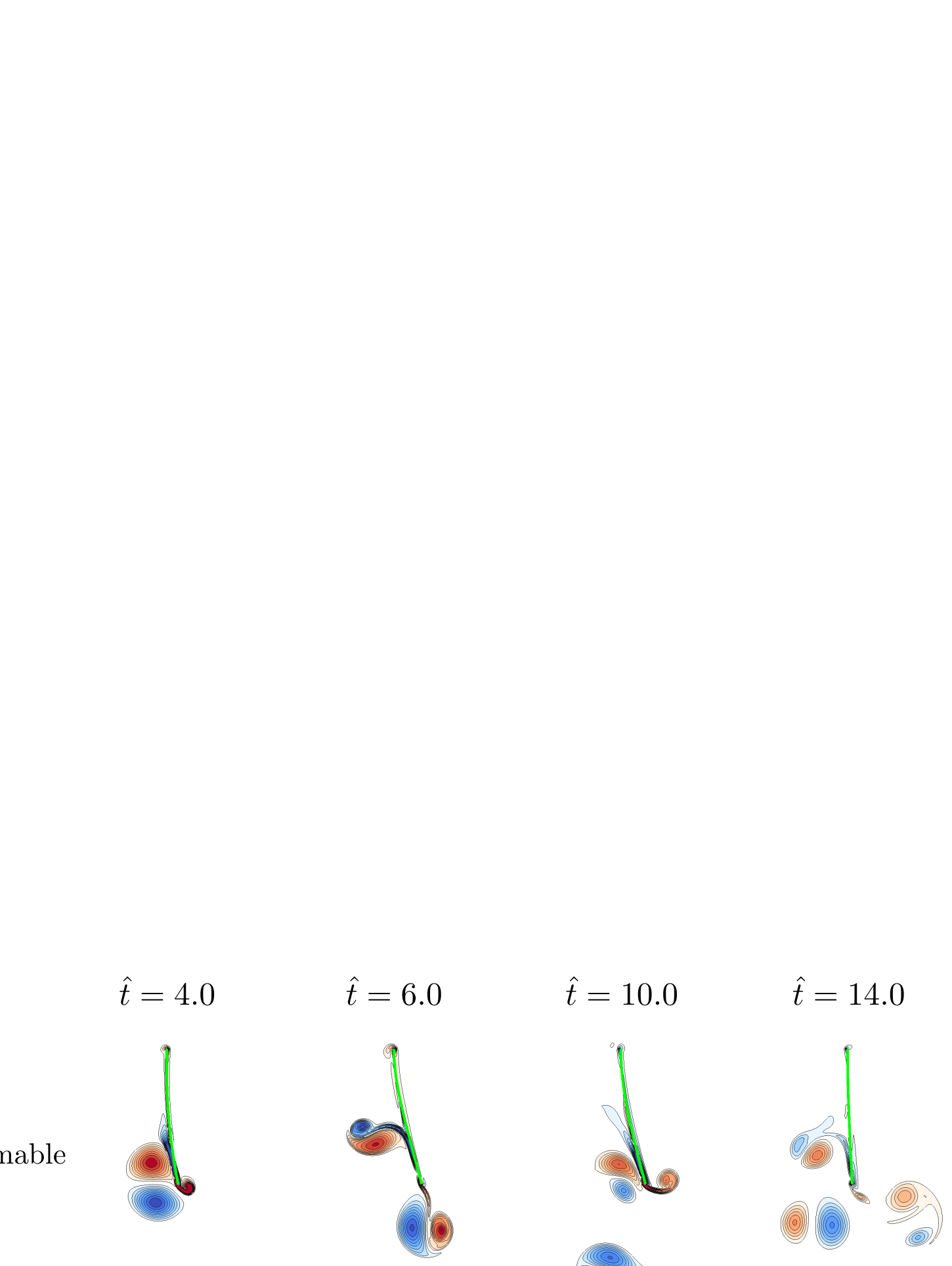

$\hat{t}=17.0$
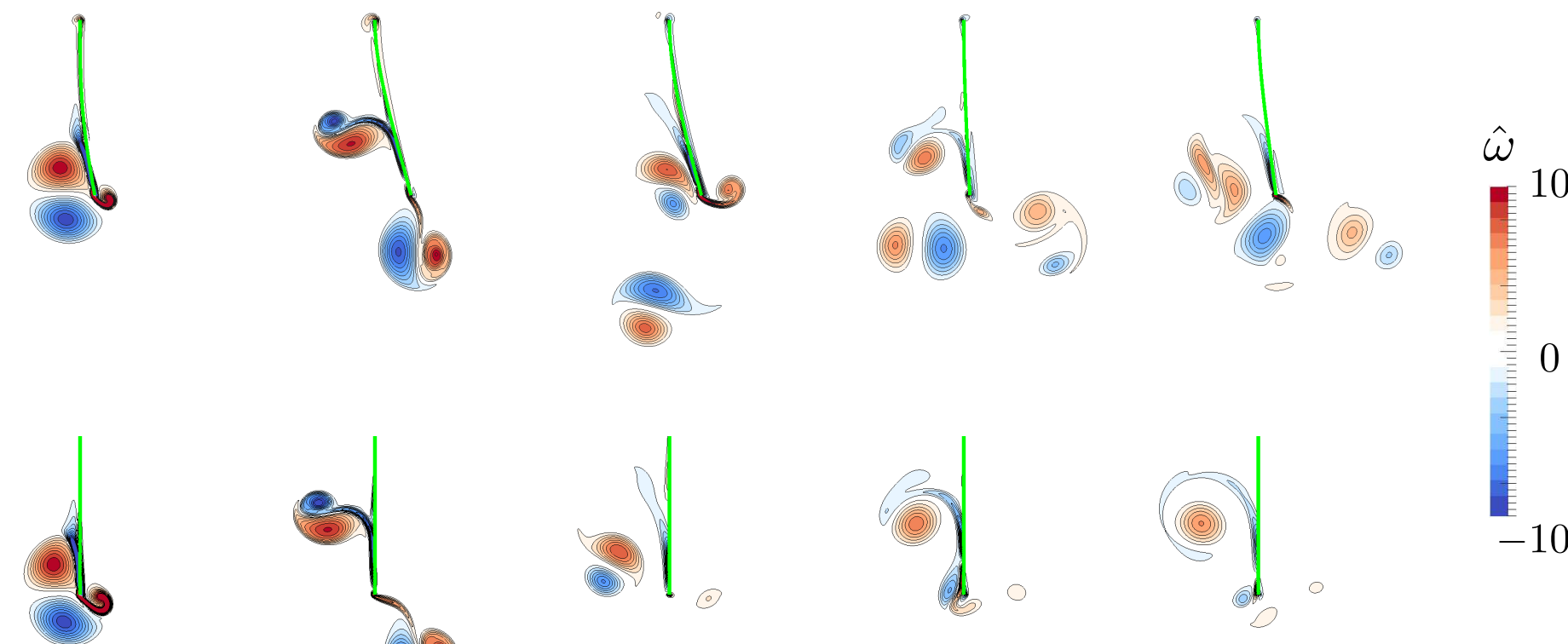

Rigid
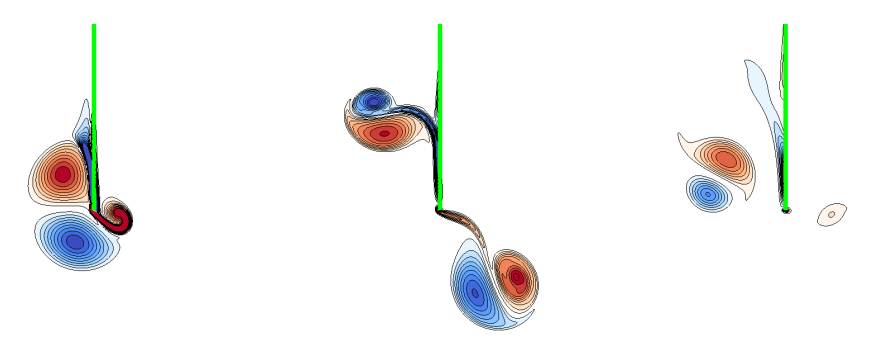

Deformable

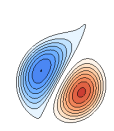

10

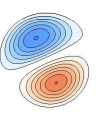



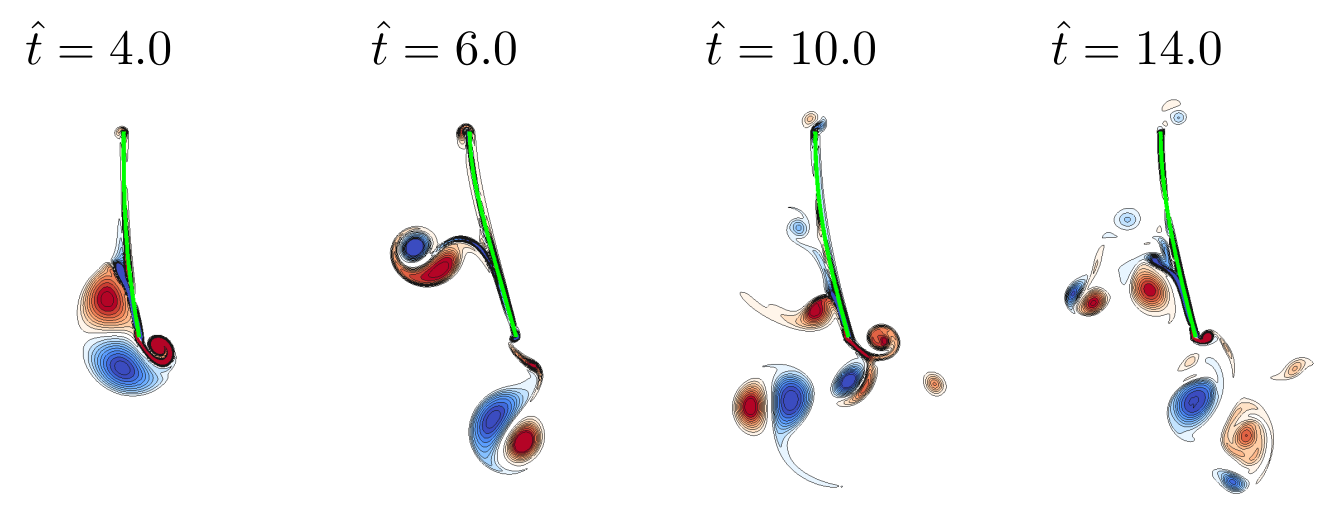

$$
\hat{t}=17.0
$$

Deformable

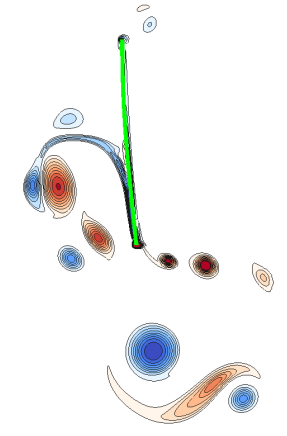

Rigid
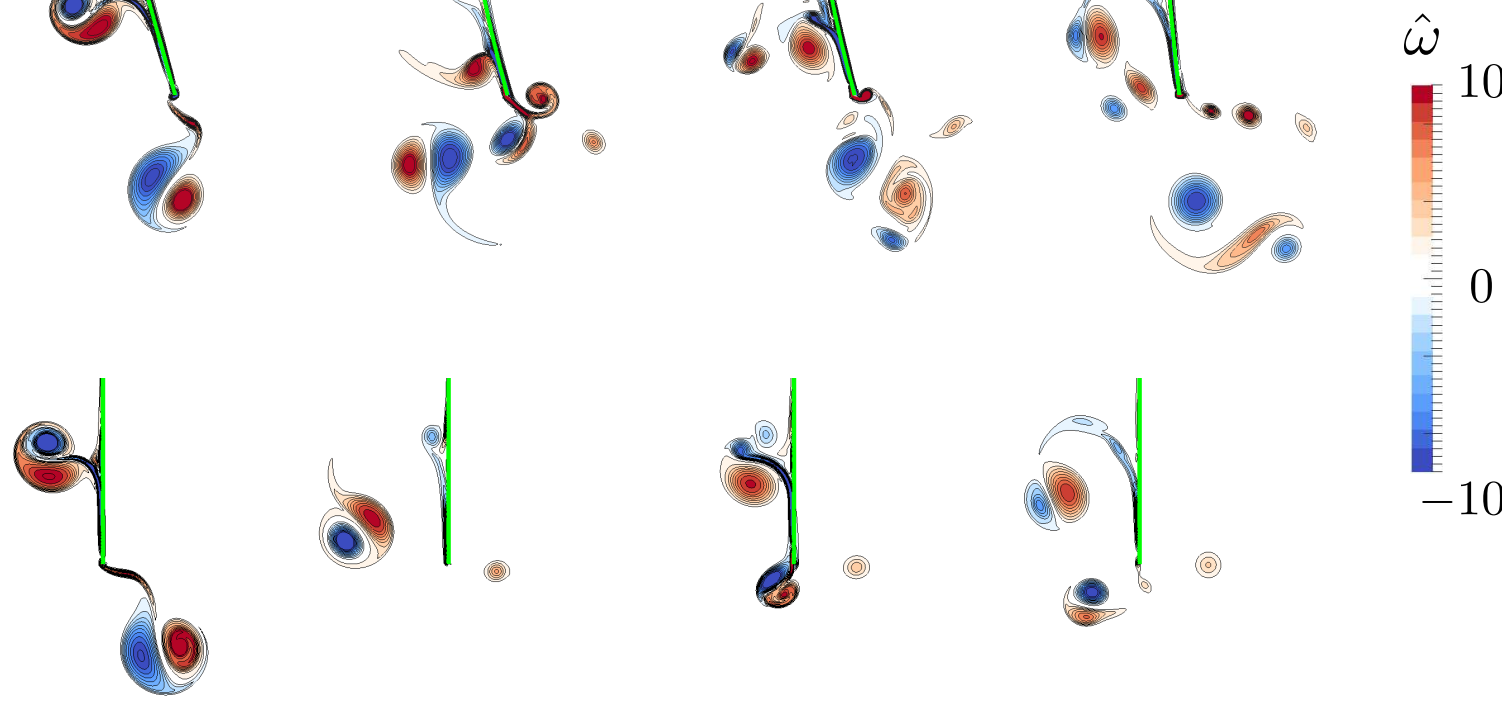
Deformable
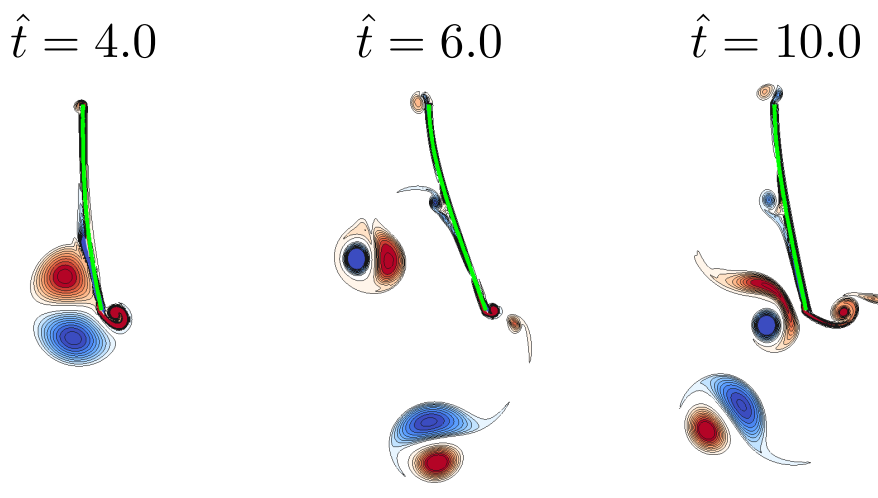

$\hat{t}=14.0$

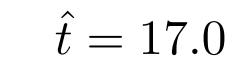

Rigid

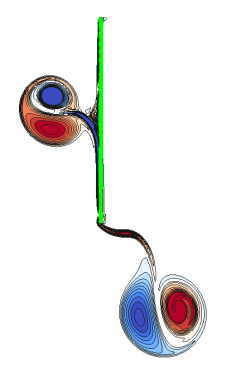

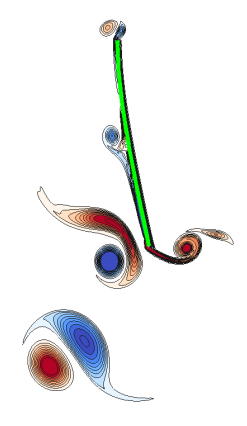
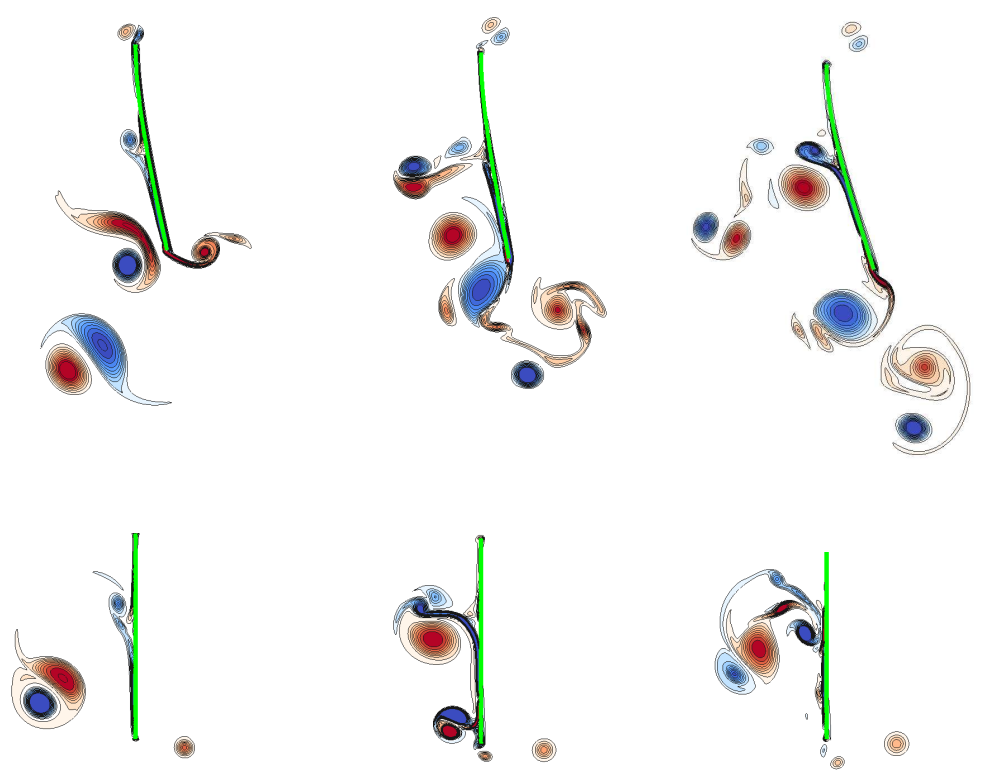
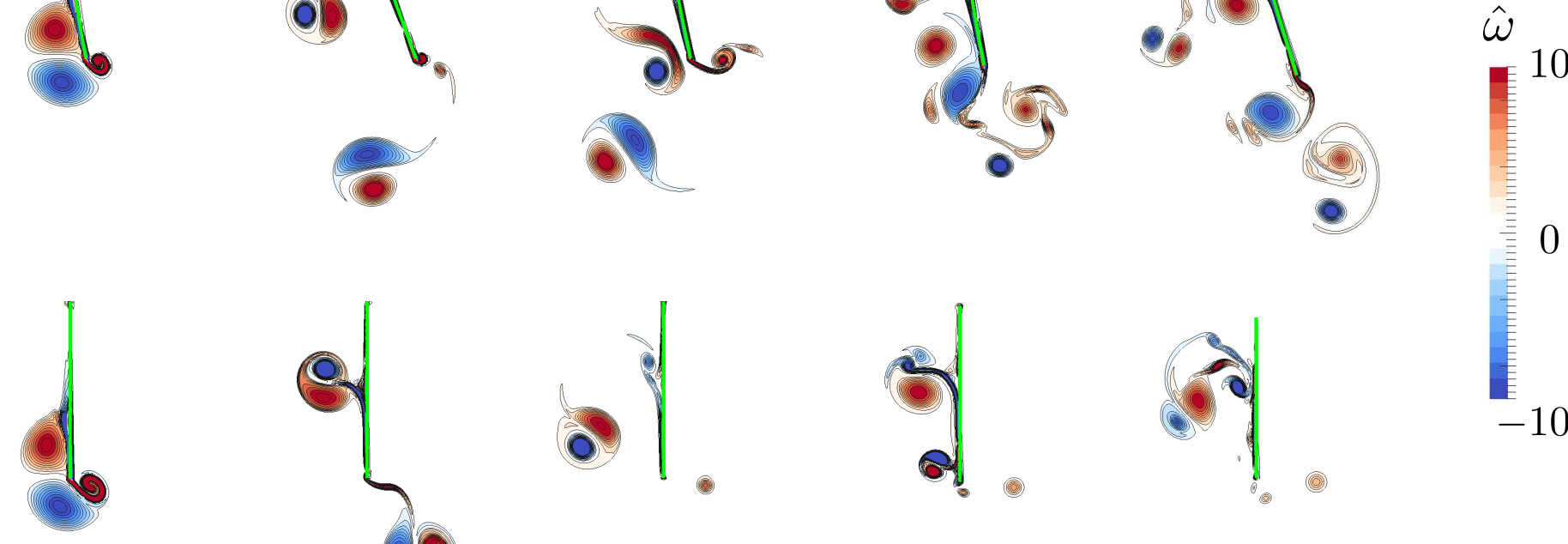


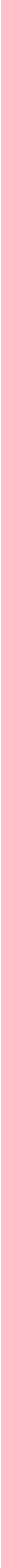

$\mathrm{Re}$

\section{Figure}

$\left|\frac{\Gamma^{+}}{\Gamma-}\right|$

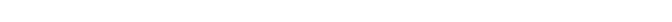
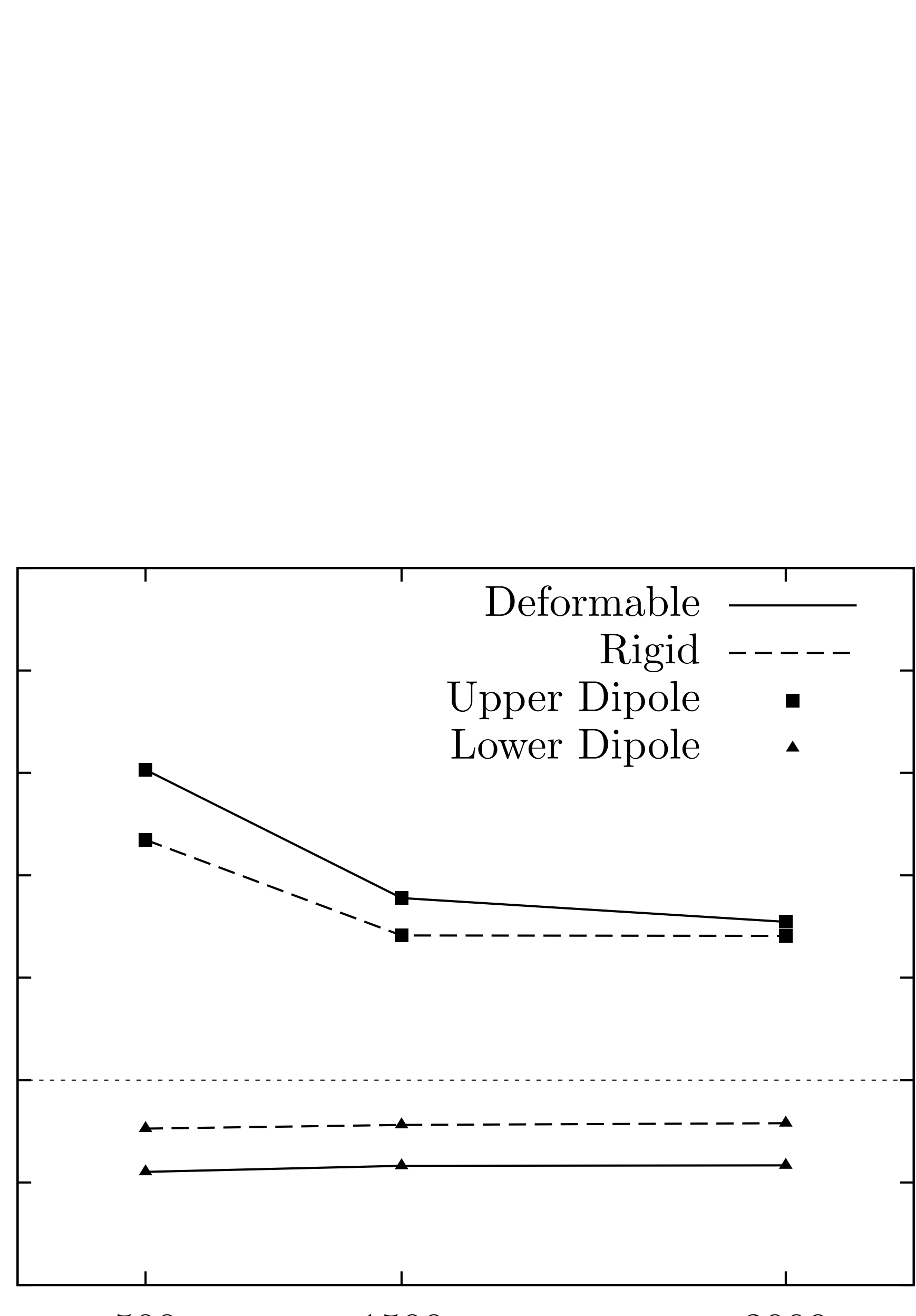
$\begin{array}{llllllllll}2.5 & 2 & 1.5 & 1 & 0.5 & 0 & -0.5 & -1 & -1.5\end{array}$
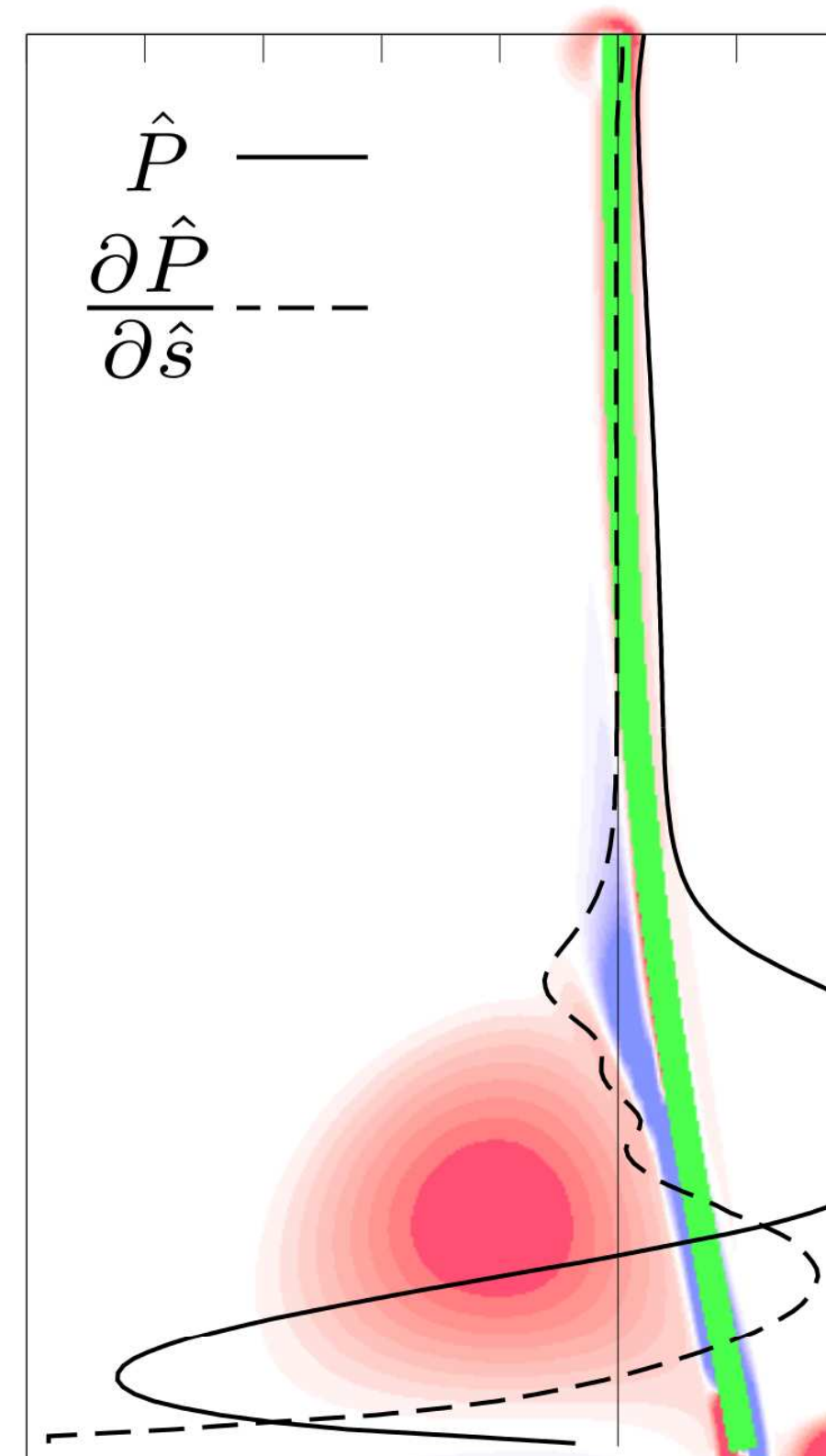

2

10

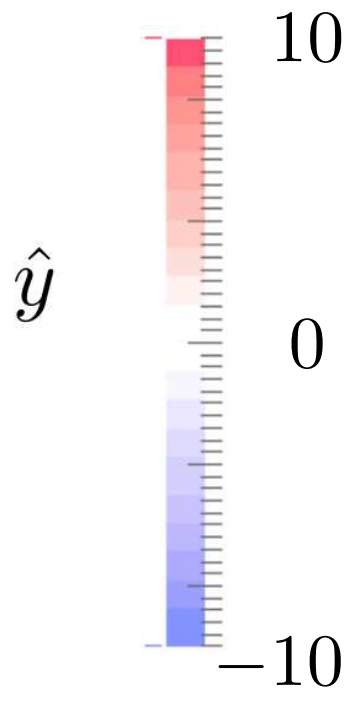

0

$-1$

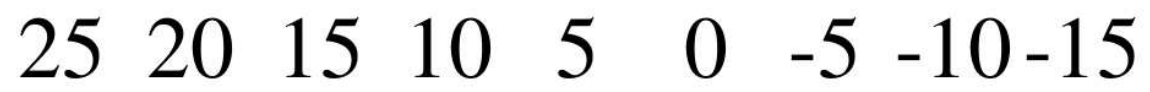

$$
\frac{\partial \hat{P}}{\partial \hat{s}}
$$




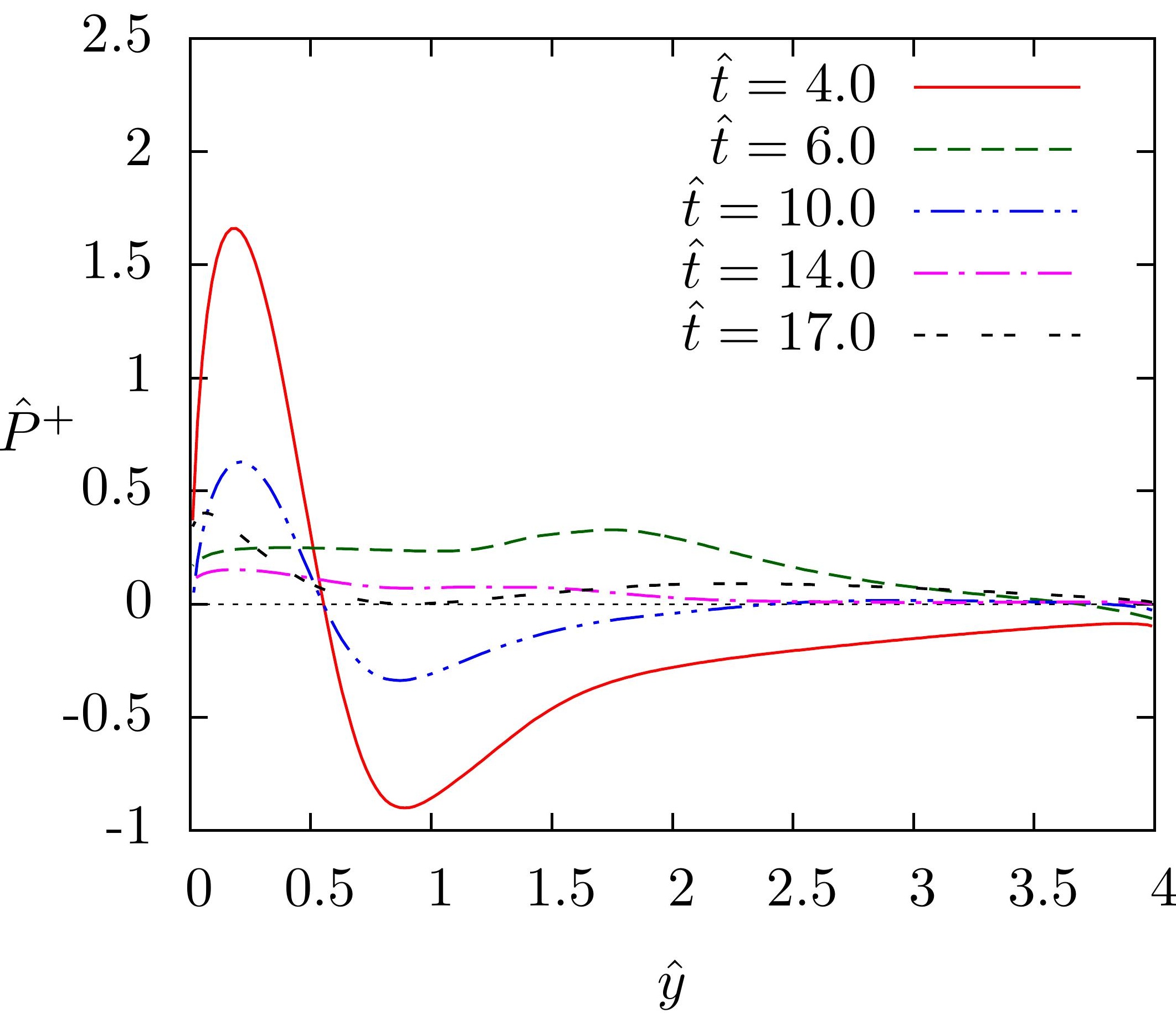




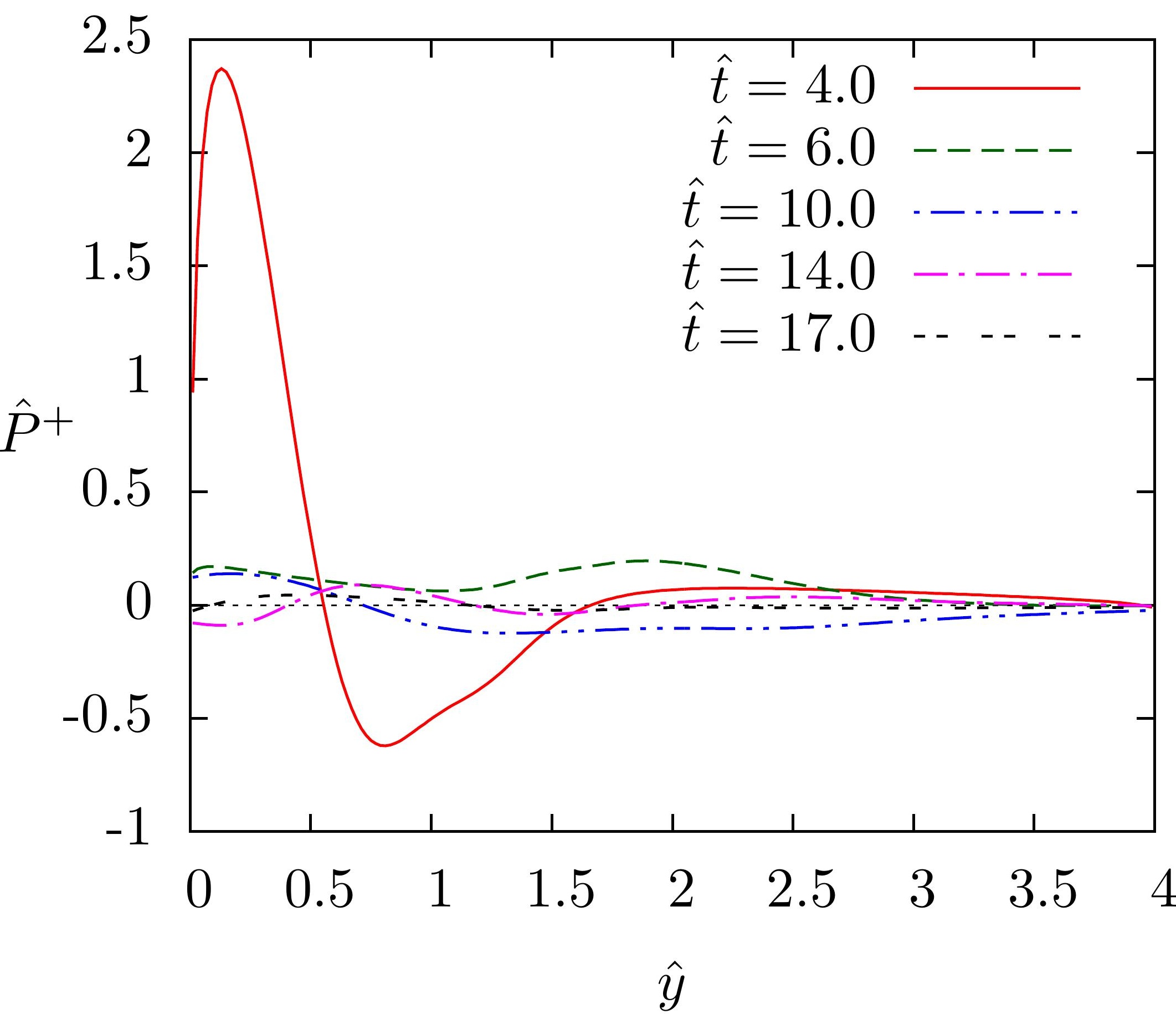




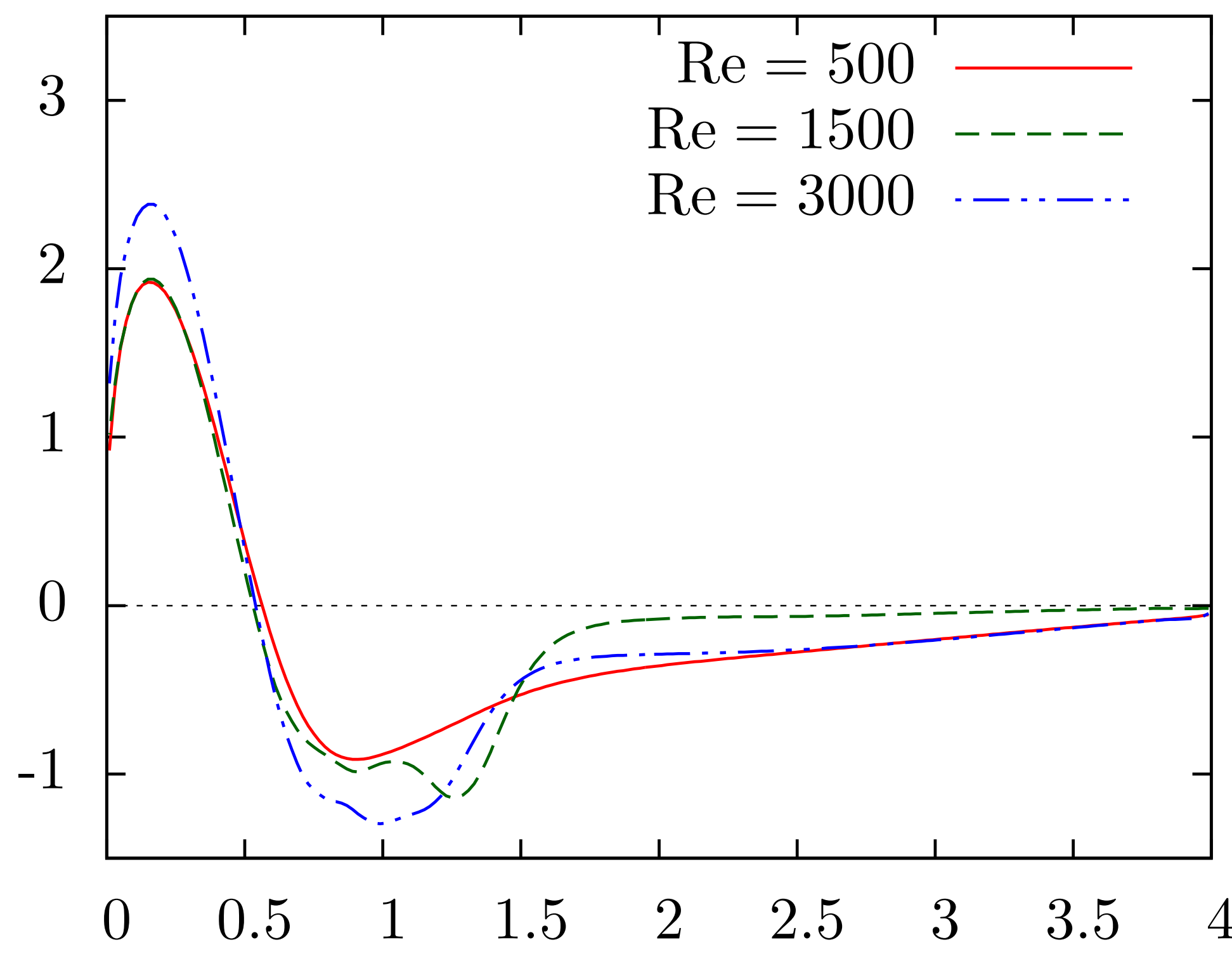




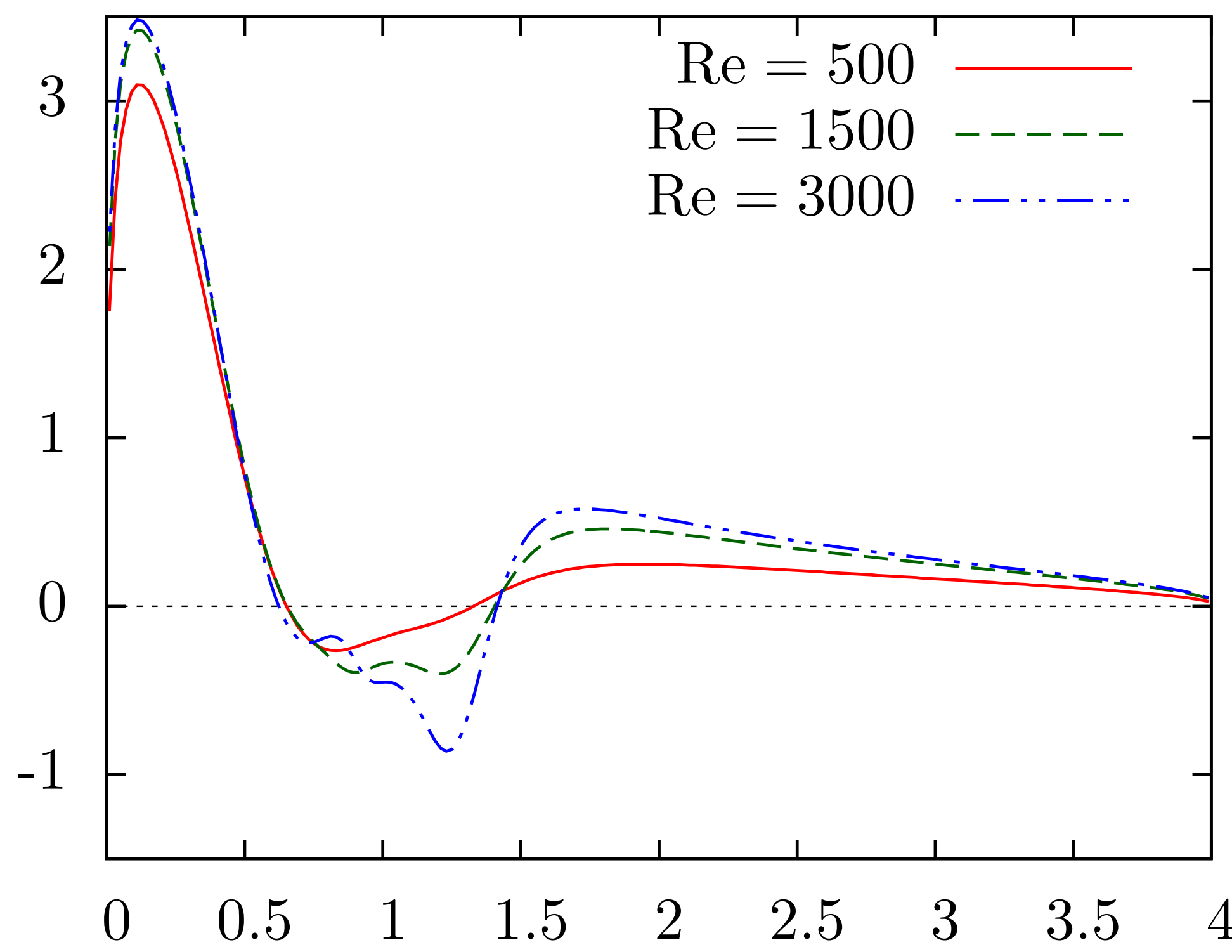




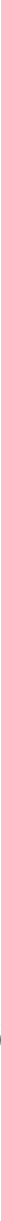

Figure

.

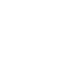
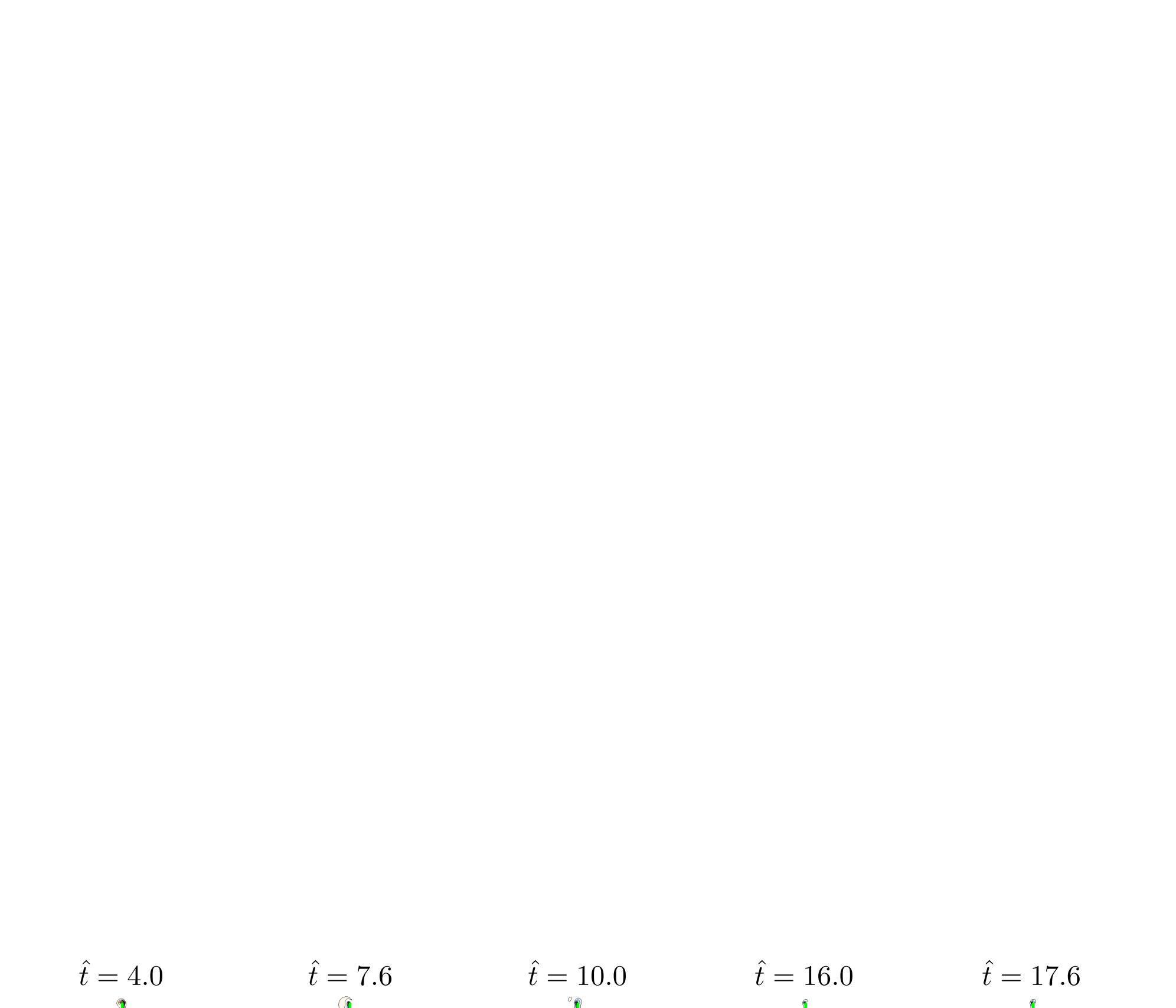


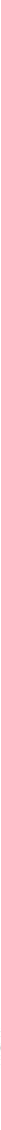

Figure 


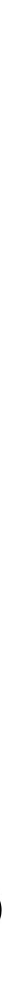

Figure 


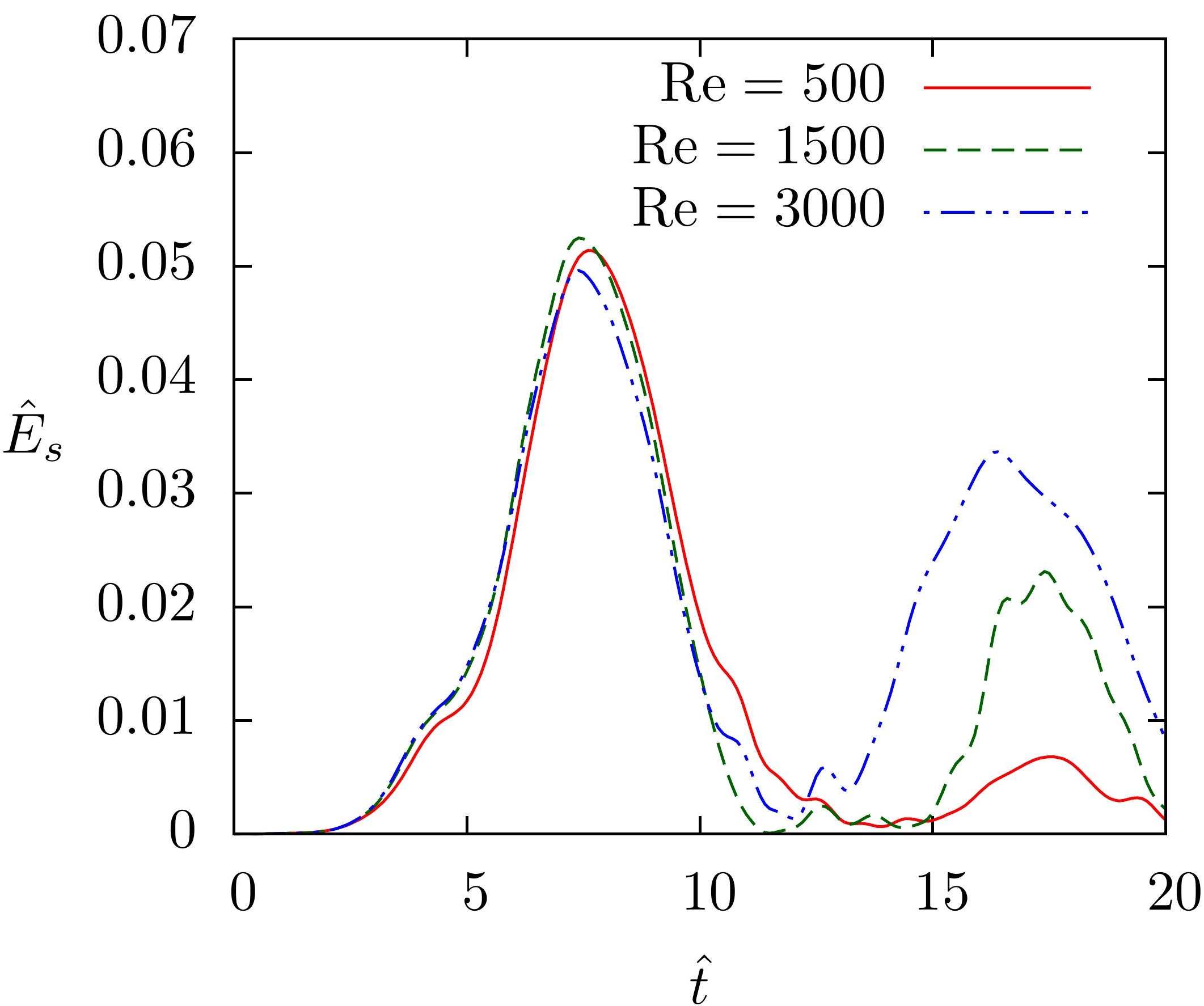




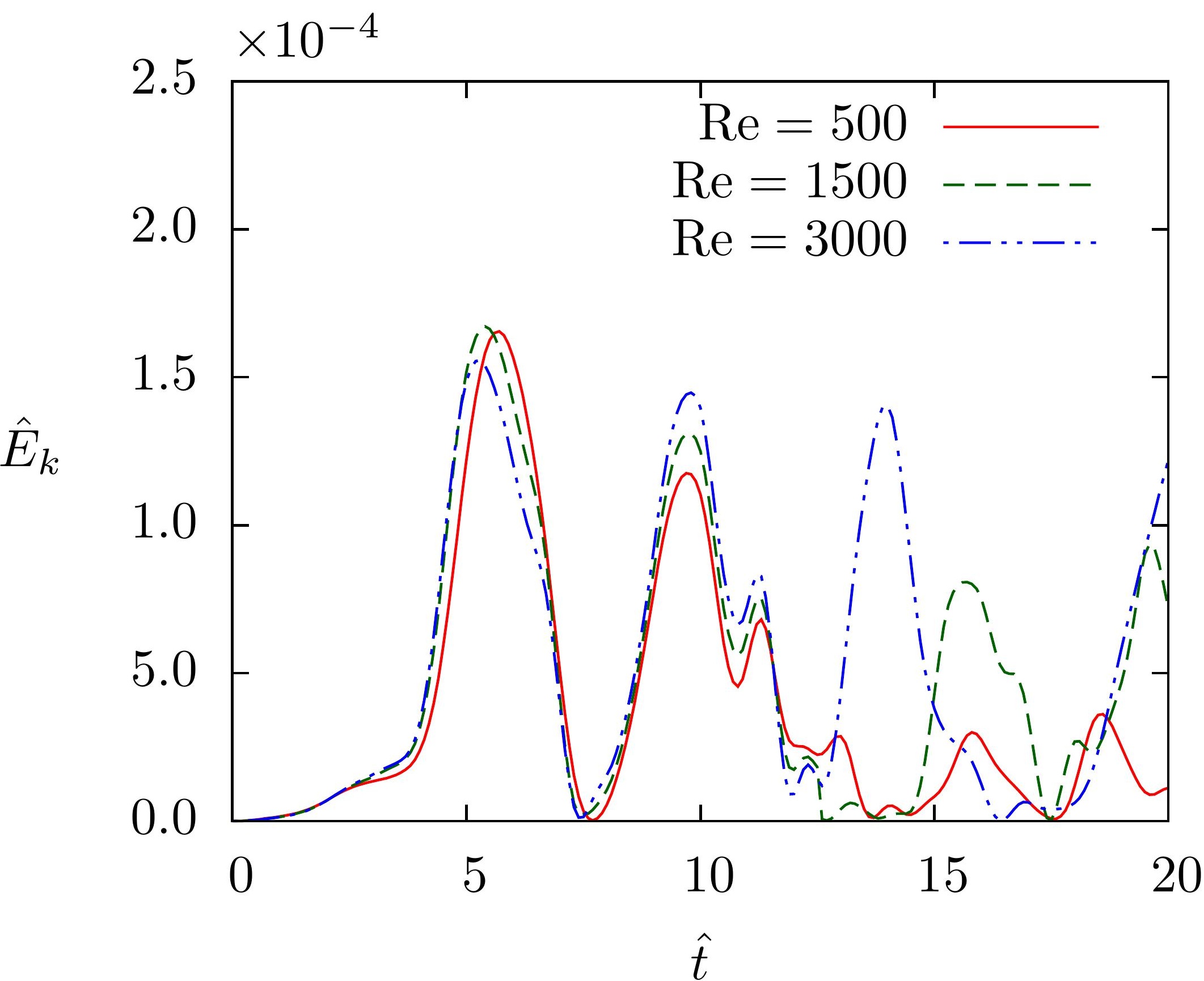

